\title{
Gold Clusters in the Gas Phase
}

\author{
Alex P. Woodham, André Fielicke* \\ Institut für Optik und Atomare Physik, Technische Universität Berlin, Hardenbergstr 36, D- \\ 10623 Berlin, Germany \\ Fritz-Haber-Institut der Max-Planck-Gesellschaft, Faradayweg 4-6, D-14195, Berlin, \\ Germany \\ fielicke@physik.tu-berlin.de
}

\begin{abstract}
Gold clusters exhibit strong size and charge state dependent variations in their properties. This is demonstrated in significant changes in their geometric structures and also in their chemical properties. Here we focus on clusters containing up to about 20 gold atoms and shortly review their structural evolution emphasizing the role of isomerism and structural fluxionality. The discussion of chemical properties is limited to the interaction of gold clusters with molecular oxygen and carbon monoxide, separately, and their interaction in $\mathrm{CO} / \mathrm{O}_{2}$ co-adsorbates on gold clusters eventually leading to $\mathrm{CO}$ oxidation. While highlighting results obtained using different experimental approaches, special attention is given to the insights obtained using infrared multiple photon dissociation (IR-MPD) spectroscopy.
\end{abstract}

Keywords gold clusters, structure, vibrational spectroscopy, reactions, carbon monoxide, oxygen, catalysis 


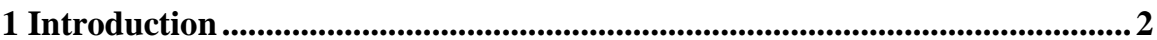

2 Experimental Methods......................................................................................... 4

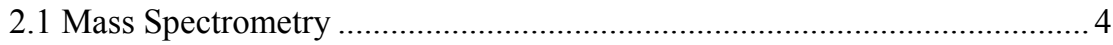

2.2 Trapped Ion Electron Diffraction …………………............................... 5

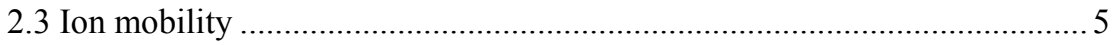

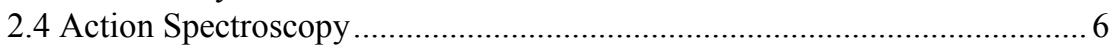

2.4.1 Anion Photoelectron Spectroscopy ………………………………...... 6

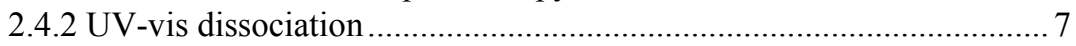

2.4.3 Infrared multiple photon dissociation (IR-MPD) …………………..... 8

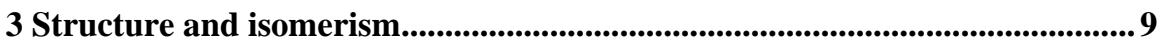

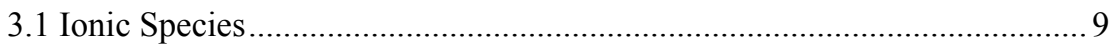

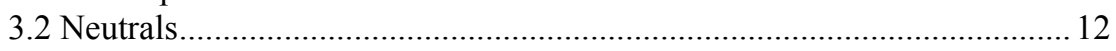

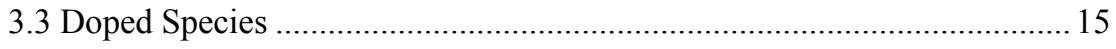

4 Gold cluster complexes ............................................................................................ 18

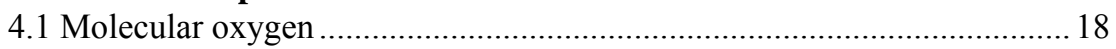

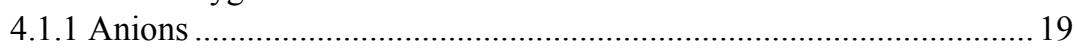

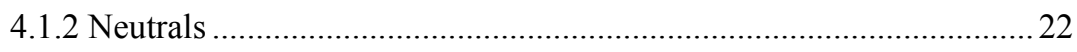

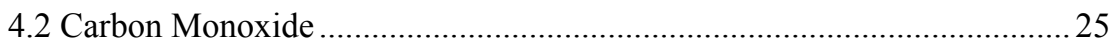

4.3 Carbon Monoxide and Oxygen................................................................ 31

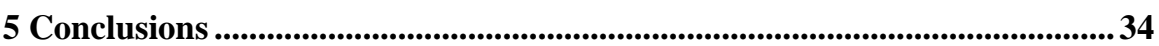

\section{Introduction}

When investigating supported gold clusters, either on a perfectly crystalline surface or a more "real" sample, the presence of the substrate represents a significant obstacle to accurate characterization. Whilst techniques do exist to overcome these difficulties, as have been detailed elsewhere in this review series, it is often advantageous to remove the clusters from the support and instead investigate them as free species in the gas phase.

Such a transition into the gas phase has several key advantages, firstly size selectivity is comparatively trivial and so property changes of the clusters can be investigated on an atom-by-atom basis. Similarly, due to the isolation afforded by working in the gas phase, ionic species can be interrogated allowing for the effects of the charge state of the cluster to be directly probed. The insights into charge state effects can be related to known charging phenomena of clusters adsorbed to surfaces [1-3]. It also provides an additional tool to probe the interplay of the electronic and geometric structures and their effects on the reactivity of the clusters, something which is known to be critically important for gold. Lastly, the transition 
to the gas phase opens up a multitude of experimental characterization techniques which are well established and understood.

Gas-phase studies have already had a marked impact on the understanding of the structures of the gold clusters. For example it has been found that small anionic gold clusters adopt a fascinating array of structural motifs; from planar structures which persist until much larger than expected cluster sizes [4-7], through to hollow cage motifs [8] and finally tetrahedral structures - like the $\mathrm{Au}_{20}$ pyramid $[9,10]$. Such structural assignments are only possible by comparison of the experimental data with theoretical predictions and here again clusters in the gas phase serve an important role: They are simplified model systems against which quantum chemical approaches can be tested for their suitability before application to more complex systems, for example particles interacting with a substrate.

In addition to these fundamental investigations of the bare cluster structures the complexes with ligands at well-defined and variable coverage, achieved through sequential addition, can also be studied. This allows for insights into reactive intermediates [11-15] and even entire catalytic reaction cycles to be mapped out [16, 17].

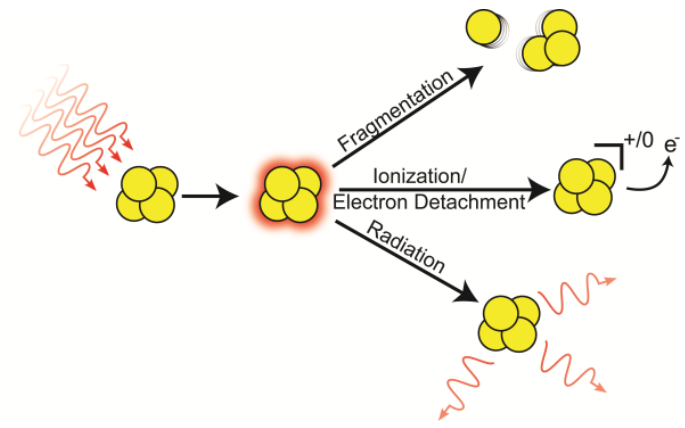

Figure 1 Schematic for energy loss pathways for an optically excited cluster.

These advantages, however, are not without their cost. Gas-phase clusters are usually not produced size-selectively but the aggregation process instead leads to a broad distribution of sizes. Thus, in most cases, experimental characterization needs to be coupled with mass spectrometric size determination or selection in order to assign properties, e.g. spectral characteristics, to a single species or cluster size. Additionally, in the case of spectroscopy, the number densities typically achievable are too low for Beer-Lambert type absorption measurements. These issues can be addressed in a variety of ways, for example applying non-optical techniques, such as electron diffraction or ion mobility, which are more direct measures of a cluster's geometric structure and sensitive to smaller quantities of substrate. Alternatively action spectroscopy can be employed where, rather than looking for the effects of matter upon light, one looks for the effects of the light upon the matter. In this way it is possible to observe processes which are free from the background signal of the probing laser pulse. Optical spectroscopy has the advantage over the more direct methods that whilst it can provide structural infor- 
mation (for example through vibrational spectroscopy) it also gives access to information concerning the electronic structure of the clusters as well (via UV-vis spectroscopies). Figure 1 shows the energy loss mechanisms available to an isolated cluster which has been excited by photon absorption and which can be exploited as markers for action spectroscopy. Of these three decay paths only the fragmentation and ionisation or electron detachment are regularly employed in gold cluster spectroscopy.

In this review we provide an overview of some of the key developments in the gas-phase studies of gold clusters, starting with a brief outline of experimental techniques, we then highlight the structural properties of gold clusters in different charge states before finally discussing the interaction of gold clusters with $\mathrm{O}_{2}$ and $\mathrm{CO}$ as prototypical reactants in the oxidation reactions which gold nano-particles are famous for. The structural information on charged gold clusters as obtained from trapped ion electron diffraction (TIED) and ion mobility experiments have recently been reviewed [18] as have the developments from anion photo electron spectroscopy (PES) [19, 20]. Finally, the chemistry of ionic gold clusters (and their binary silver-gold and pure silver analogues) have also been discussed elsewhere [21]. These reviews focus on the charged gold clusters and surprisingly large differences in, for example, their geometric shapes and the size for the 2D3D transition have been found between the charge states. Such a dramatic influence of charge state raises intriguing questions concerning the intermediate case, that of the neutral clusters, which shall be discussed in more detail here.

\section{Experimental Methods}

\subsection{Mass Spectrometry}

Conceptually one of the simplest experimental techniques applied to clusters, and indeed the basis of most gas-phase studies, is mass spectrometry. Very different techniques for mass analysis have been applied to separate and/or trap charged clusters, for example classical electric or magnetic sector-field instruments, timeof-flight measurements, quadrupole mass filters, and Paul or Penning traps. Mass spectrometry is indispensable in the analysis and control of cluster formation in cluster sources where typically a supersaturated atomic vapour aggregates into a distribution of cluster sizes. By now this field of mass spectrometric characterization of clusters is very well established and so we shall refrain from discussing further technical details here. It should be noted that mass spectrometry requires the presence of charged clusters, however, by employing suitable ionisation techniques prior to mass analysis, information on the neutral clusters may also be obtained. Despite its conceptual simplicity a great deal of information can be ex- 
tracted from the mass analysis of a cluster distribution, concerning relative stabilities of different clusters (cf. 'magic' sizes) and the reactivity of the clusters.

In addition to this kinetic reaction studies can also be performed, i.e. analyzing the products formed with changing reaction times or reactant partial pressure. Knowledge of the kinetics allows for the individual steps in the reaction paths to be modelled and ultimately can lead to the elucidation of complete reaction mechanisms $[16,17]$.

\subsection{Trapped Ion Electron Diffraction}

One approach to investigating cluster structures is trapped ion electron diffraction (TIED) [22, 23]. In TIED an electron beam of several tens of $\mathrm{keV}$ is directed at a sample of size-selected clusters held in an ion trap. The electrons are diffracted by the atoms and the resulting diffraction pattern is recorded, typically with a CCD camera. The radial variance in the electron intensity pattern is then compared with simulated curves and structural assignment is made based upon the best agreement between theory and experiment. Experimentally the cluster sizes which can be investigated are limited at the lower end by the atomic scattering intensity and at the high end by the mass resolution of the experiment. In practice this leads to a size range of $\mathrm{Au}_{n} n=10-200$ being experimentally tractable [18] and, owing to the trap requirements, is limited to charged clusters. Further, as the high energy electron beam can induce ionisation and fragmentation, often anionic species are preferred as they result in neutral products, which are then lost from the trap and so do not complicate the recorded radial distribution function.

\subsection{Ion mobility}

Another non-optical approach to structural determination of clusters is the measurement of their collisional cross sections via ion mobility [24]. Charged clusters are injected into a drift cell filled with a pressure of inert gas (typically He) and an electrostatic potential gradient applied across it. The ionic clusters are then guided towards the far end of the drift cell by this potential and their arrival times are measured. Those clusters with a larger cross section experience more collisions with the buffer gas and thus a greater resistance to their motion, resulting in a longer drift time. Again candidate structures obtained from quantum chemical calculations can be used to generate theoretical collisional cross sections which are compared with experiment, resulting in structural assignment. Unlike TIED, ion mobility measurements are not limited at a smaller size but differences in collisional cross sections for structural isomers become increasingly marginal as clusters become larger, effectively limiting the upper size which can be investigated. 
Obviously as the clusters are accelerated with an electrostatic interaction, this also can only be applied to charged species.

\subsection{Action Spectroscopy}

Optical spectra can be recorded by using action spectroscopy to overcome the limitations of transient absorption (Beer-Lambert type) spectroscopy. As has been mentioned, action spectroscopy reverses the traditional paradigm of looking at the effects of matter on light by looking for changes in the clusters themselves as the marker for optical absorption. By performing these spectroscopies in the UV-IR frequency range one is able to directly probe both the geometric (IR) and electronic (UV-vis) structures of the clusters.

\subsubsection{Anion Photoelectron Spectroscopy}

For metal clusters, one of the most commonly applied action spectroscopies is anion PES. In this variant of photoemission spectroscopy [25] the anionic clusters are mass selected, allowing for insights into the evolution of the internal electronic structure as the clusters grow atom-by-atom [26]. Typically, UV-vis photons are used to detach electrons from the anionic clusters and the resulting kinetic energy distribution of the detached electrons is recorded. The observed distribution is governed by the energy balance equation:

$$
K E_{e^{-}}=h v-B E+E_{\text {int }, I}-E_{\text {int }, F}
$$

where $\mathrm{KE}_{\mathrm{e}-}$ is the electron kinetic energy, $\mathrm{h} v$ the photon energy, $\mathrm{BE}$ the binding energy of the electron (=-Electron Affinity (EA)) and $E_{\text {int }}$ is the combined internal electronic, vibrational and rotational energy of the initial (I) and final (F) states respectively. When starting with an anionic cluster in its ground state, i.e. $\mathrm{E}_{\text {int, } I}$ equals 0 , the resulting distribution for the kinetic energy of the electrons reflects the internal energy contained within the final structure, with the fastest electrons corresponding to the EA of the cluster. Alternatively electron detachment may occur from electronically or vibrationally excited states of the anion giving even more detailed information on the electronic and geometric structures of the observed clusters. For example the observation of vibrational hot bands provides access to the vibrational constants of the anionic state. It should be noted that, as with all spectroscopies, there are selection rules which govern the observable transitions and in the case of anion photoelectron spectroscopy the observed transitions are governed by the Franck-Condon factor [27]. 
Typically photon energies far in excess of the EA (UV photons) are used and the resulting spectra are compared to the calculated EAs, excitation energies, and/or electronic density of states from trial structures, allowing for structural assignment of the anionic structure. In such a regime the effects of the vibrational energy can mostly be ignored as $E_{\text {elec }}>>E_{\text {vib }}$. An alternative scheme, utilising the principles of ZEKE and SEVI [27] where photons much closer to threshold detachment are used, allows for greater absolute energy resolution and thus vibrational progressions are more regularly recorded and lower frequency vibrational progressions are theoretically accessible. Such vibrational information may also then be compared with theoretical modelling allowing for structural assignment [28].

There are, however, some considerations which must be borne in mind when using anion PES. Firstly, whilst the technique is in principle sensitive to both the anionic and neutral states of the cluster, because the photodetachment is considered to be a prompt process, that is, fast on the time scale of nuclear motion, it actually gives information on the neutral cluster in the geometry of the anion. This can have consequences when concluding about neutral cluster properties based on PES results, particularly because the structures of gold clusters can vary significantly with the charge state $[18,29]$. Secondly, the vibrational selection rules for anion PES are such that generally only the transitions between totally symmetric vibrations are allowed and this somewhat limits the utility of vibrationally resolved anion PES.

\subsubsection{UV-vis dissociation}

An alternative method using ultraviolet/visible (UV-vis) photons is to induce fragmentation, typically of complexes formed between the cluster of interest and a rare-gas "tag". Through monitoring of the mass spectrometric intensities, changes induced by the UV-vis photons allow for the electronic excitation spectrum of the clusters to be recorded, which again may be compared to theory and leads to a structural assignment. For a truly accurate understanding one needs knowledge of both the ground state and the excited state properties, which are often computationally very demanding to calculate [30-32]. Perhaps owing to the additional computational difficulties associated with electronic spectroscopies the number of investigations utilizing this technique for gold clusters is somewhat limited [3135]. These investigations reveal the strong relativistic effects in gold clusters, borne out in strong $s-d$ hybridization, and is given as the cause for both the relatively complicated optical spectra observed, and the failure of the plasmon model, as extended from bulk properties, in predicting the observed spectra. An alternative approach to such spectra is the investigation of mass selected neutralized clusters in rare gas matrices [36].

A further modification to this dissociation spectroscopy is to record the resultant kinetic energy distributions of the fragments using the VMI technique. This al- 
lows for more detailed information concerning the excited states of the clusters and the energy partitioning between the different fragments to be elucidated. Currently, however, this technique has been limited in its application to the gold-rare gas hetero-dimers $[37,38]$.

\subsubsection{Infrared multiple photon dissociation (IR-MPD)}

Whilst UV-vis excitation probes electronic transitions, vibrational transitions can similarly be excited by using infrared light. The vibrational spectrum of a cluster is a sensitive fingerprint of its geometric structure. As the energy of an individual photon in the mid- and far- IR is significantly reduced compared to that in the UV-vis range, inducing ionization, electron detachment, or fragmentation usually requires absorption of very many IR photons by a single cluster [39-41]. One way of reducing the number of photons needed to induce a mass spectrometrically detectable action is the application of the "messenger tagging" method. In this variant an atom or molecule is weakly bound to the surface of the cluster, introducing a readily fragmentable bond into the system. Thus when sufficient energy is deposited into the cluster via IR absorption this bond breaks resulting in a change in mass, serving as a "messenger" for the absorption. Depending on the messenger and the frequency of the probed vibrational mode the binding energy may be sufficiently low to be sensitive to the absorption of single IR photons.

For many metal clusters the vibrational fundamentals lie below $400 \mathrm{~cm}^{-1}(\sim 50$ $\mathrm{meV})$. Despite the weak bonding in the cluster system fragmentation of messenger complexes still usually requires absorption of multiple photons. This, coupled with the low IR absorption cross sections of metal clusters, necessitates the use of an intense, widely tunable IR source. At present the only method for generating sufficiently intense IR pulses at these wavelengths is to use IR Free Electron Lasers (FELs) and so far all these experiments on gas-phase clusters have been performed at the Free Electron Laser for Infrared eXperiments facility (FELIX) in the Netherlands [42]. To date, FEL based IR-MPD has been applied to investigate the structures of a wide range of transition metal clusters, more explicit details of the experiment can be found elsewhere [43, 44].

In addition to using rare-gas atoms as messengers for the IR absorption it is possible to instead rely on the dissociation of a complex with a ligand of chemical relevance, e.g. $\mathrm{CO}$ or $\mathrm{O}_{2}$ as will be discussed later on. This has the further advantage of introducing a stronger (typically several orders of magnitude) chromophore into the cluster, facilitating the IR-MPD process. 


\section{Structure and isomerism}

\subsection{Ionic Species}

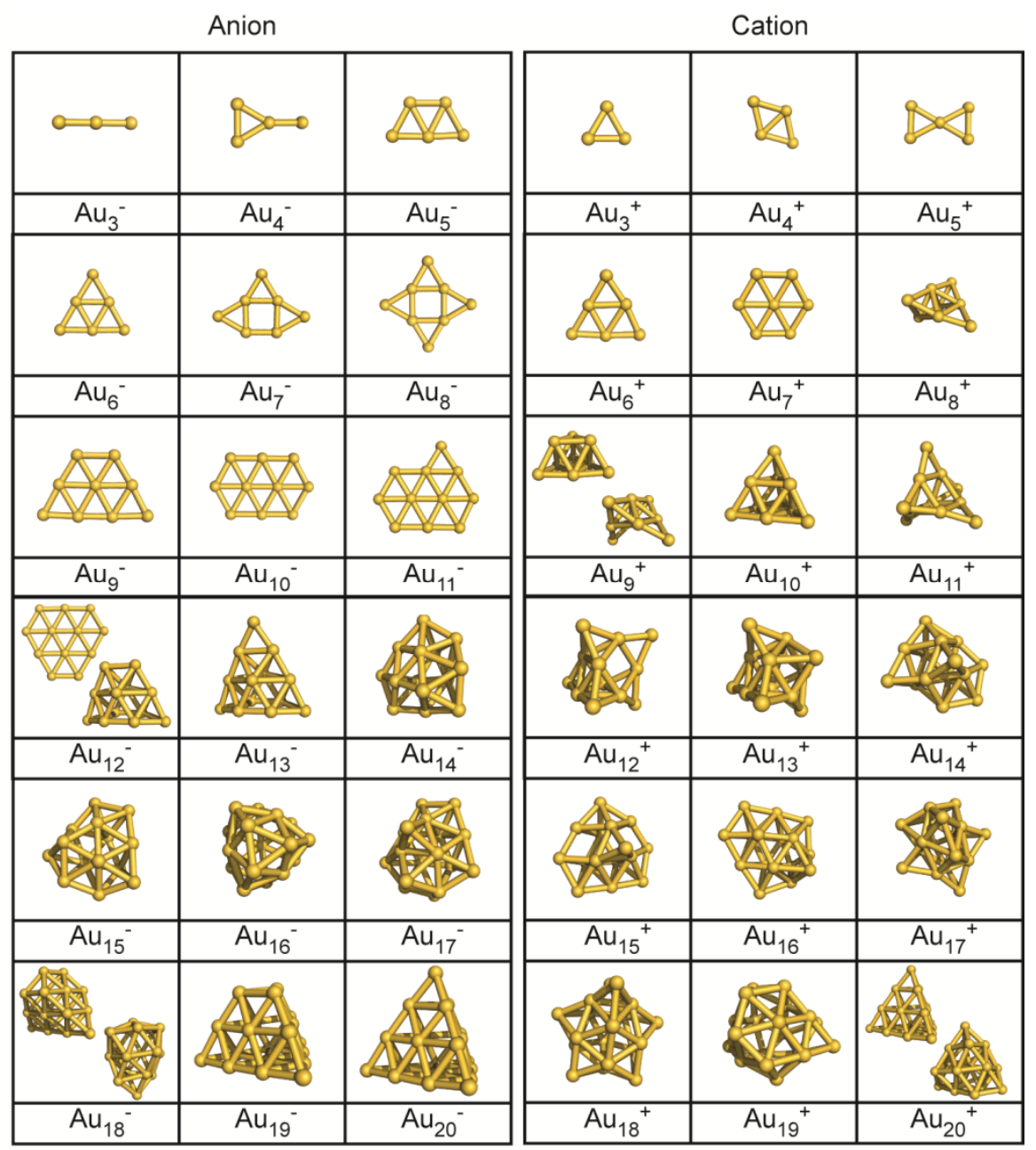

Figure 2: Structures of anionic and cationic gold clusters, structures are taken from Ref. [18]

Initial experimental investigations into the structures of free, bare gold clusters focused on the ionic species and a summary of the structures identified based on ion mobility and electron diffraction studies [18] is reproduced in Figure 2. The small gold clusters remain two-dimensional until, at a certain critical size, begin to form double-layered or three-dimensional structures (as opposed to polyhedral 
motifs). This structural pattern has been attributed to the relativistic effect leading to a strong $s-d$ hybridization (as was also observed from UV-vis spectroscopy, see earlier) [45-47]. It is interesting to note the substantial differences between the structures of the anions and cations - the anions remaining 2D until much larger cluster sizes than their cationic counterparts. Whilst this is the most prominent structural deviation, other significant differences can also be observed between the larger clusters. Figure 2 also clearly demonstrates the existence of structural isomers for some of the cluster sizes. Recently more cases of structural isomerism or fluxionality have been found and the implications of this for the observed reactivities have been noted,
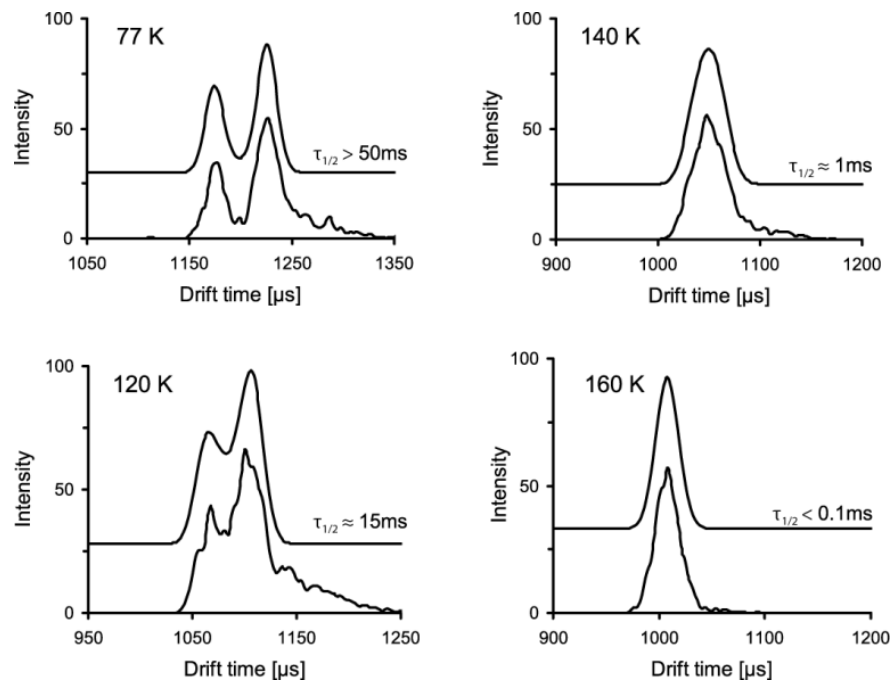

Figure 3: Arrival time distribution for $\mathrm{Au}_{9}{ }^{+}$as a function of drift cell temperature. At low temperature two isomers are detected, while at elevated temperature their rapid inter-conversion leads to only a single peak in the drift-time distribution. See Figure 2 for the structures of the two isomers. Reprinted with permission from Weis, P., et al. (2002) J Chem. Phys. 117: 9293. Copyright 2002, AIP publishing LLC

The first direct evidence for structural isomerism in gold clusters came from the ion drift studies of both the cations and anions [4, 48, 49]. For the anions a bimodal distribution was observed in the arrival time of $\mathrm{Au}_{12}{ }^{-}$which was interpreted as due to two isomers being present, one $2 \mathrm{D}$ and one $3 \mathrm{D}$, indicating $\mathrm{Au}_{12}{ }^{-}$as the critical size for this transition. The presence of both a $2 \mathrm{D}$ and a $3 \mathrm{D}$ isomer for $\mathrm{Au}_{12}{ }^{-}$has been confirmed from multiple experiments $[4,7,50]$. The critical size for the $2 \mathrm{D}$ to $3 \mathrm{D}$ transitions, however, remains a contentious issue because of discrepencies with the corresponding theoretical calculations [51-55]. The presence of isomers and their inter-conversion was also observed for $\mathrm{Au}_{9}^{+}$[48]. When the ion drift experiment was performed at reduced temperatures what had initially been a single feature in the arrival time distribution resolved into two separate 
peaks, as shown in Figure 3. Such behaviour indicates that the cluster is undergoing rapid isomerization between two structural forms at elevated temperatures and upon cooling the barrier to inter-conversion becomes insurmountable and so the isomers become trapped.

Further insights into the importance of isomerism have been obtained from anion PES. For example in the photoelectron spectrum of $\mathrm{Au}_{10}{ }^{-}$several weaker features at low electron binding energy (BE) were observed and ultimately assigned to 3 additional isomers [56]. In this particular case the separation was achieved by exploiting the cluster's varying reactivity towards molecular oxygen allowing for the isomers to be removed from the molecular beam to leave only the ground state species (which is unreactive towards $\mathrm{O}_{2}$ ). The 4 isomeric structures and their relative energies are given in Figure 4.

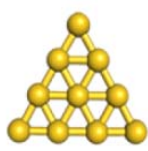

$0.00 \mathrm{eV}$

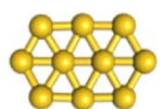

$0.16 \mathrm{eV}$

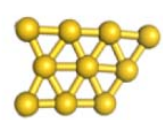

$0.18 \mathrm{eV}$

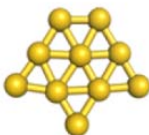

$0.14 \mathrm{eV}$

Figure 4: The four isomers of $\mathrm{Au}_{10}{ }^{-}$detected using anion PES and their relative energies to the ground state $\mathrm{D}_{3 h}$ structure

Similar investigations for the gold clusters $\mathrm{Au}_{7}{ }^{-}-\mathrm{Au}_{18}{ }^{-}$have also led to several isomers being identified, either by exploiting their different reactivity with $\mathrm{O}_{2}$ and Ar or through isoelectronic substitution. Ar binding is stronger to planar surfaces (see later) and thus Ar tagging is particularly sensitive when there is a significant change in the geometrical structure between the two competing isomers, e.g. 2D vs. 3D or cage vs. non-cage (cf. the planar faces of the pyramidal structures) [10, 57]. Similarly the use of $\mathrm{O}_{2}$ as a reactive tag is limited because the odd-numbered clusters are completely unreactive with $\mathrm{O}_{2}$ (see later). This led to the slightly more elaborate technique of isoelectronic substitution, i.e. exchanging a gold atom with a silver or copper atom. For example with $\mathrm{Au}_{7}{ }^{-}$and $\mathrm{Au}_{8}{ }^{-}$such a substitution does not greatly affect the geometric structure, but does alter the relative energies, and thus populations, of the different isomers allowing for the observed PES to be deconvoluted into the contributions arising from the different isomeric forms [5759].

This wealth of information concerning the isomers of the anionic clusters is not matched for the cationic species. The apparent lack of structural isomerism for most cationic gold clusters may be an artefact of the methodology used to probe their structures. Specifically, because the second ionization energies of gold clusters are prohibitively high for table top laser systems, a direct analogue to anion PES is not possible, and instead the majority of the structural information comes from TIED and ion drift measurements. The latter is not very sensitive to small structural changes which do not alter the collision cross section significantly, while TIED requires the presence of a considerable fraction of the isomer in order 
to resolve the different structures. Furthermore, these techniques occur on a timescale much longer than anion PES (which is almost always assumed to be a prompt photoemission process) and so if the structural isomerism is connected with rapid inter-conversion (fluxionality) it may be averaged over in the experiments.

\subsection{Neutrals}

Through the efforts of the above research relying on TIED, ion mobility measurements and anion PES a comprehensive understanding about the structures of the charged gold clusters has been achieved. Comparatively little experimental work, however, has been done on the neutral clusters. The origin of this discrepancy is eminently clear: Experimentally the above techniques do not work, nor are they easily adapted to work for neutral systems. All of them require the interaction of the charge on the particles with external fields to allow for the clusters from the nascent distribution to be resolved by size, either in time or space. Once separated in this way they can then be spectroscopically addressed on a species by species basis.

Dissociation spectroscopy, as described above, can be performed on a nonmass selected molecular beam containing charged and neutral species. As the irradiation of the cluster beam is done prior to mass analysis, it is possible to add an ionisation step after the spectroscopic probe, allowing for the recording of massspecific spectra for the neutral species. Figure 5 and Figure 6 show far-infrared spectra of $\mathrm{Au}_{7}, \mathrm{Au}_{19}$ and $\mathrm{Au}_{20}$ measured with krypton as a messenger [29]. $\mathrm{Au}_{n} \mathrm{Kr}$ complexes are formed at $100 \mathrm{~K}$ by seeding the $\mathrm{He}$ gas with a few per cent of $\mathrm{Kr}$ and they are then interrogated using the IR-MPD technique as outlined in the methods section. Ionisation is performed using $7.9 \mathrm{eV}$ photons ( $\mathrm{F}_{2}$ laser). Structural assignments become possible only by comparison with IR spectra predicted from quantum chemical calculations.

For $\mathrm{Au}_{19}$ and $\mathrm{Au}_{20}$ the harmonic vibrational spectra calculated using density functional theory within the gradient-corrected approximation allow for unequivocal assignment to pyramidal structures [29]. Neutral $\mathrm{Au}_{20}$ has the same tetrahedral structure, comprising fcc-like packing with four (111) faces of $\mathrm{Au}$, as found for the corresponding anion [9] and observed for one isomer of the cationic species [60]. Similarly, $\mathrm{Au}_{19}$ is isostructural to its anion [10]. The infrared spectra rather elegantly demonstrate the loss of symmetry upon removing a single gold atom from the $\mathrm{T}_{d} \mathrm{Au}_{20}$ to the $\mathrm{C}_{3 v} \mathrm{Au}_{19}$ cluster leading to a splitting of the single intense peak in $\mathrm{Au}_{20}$ into two signals. 


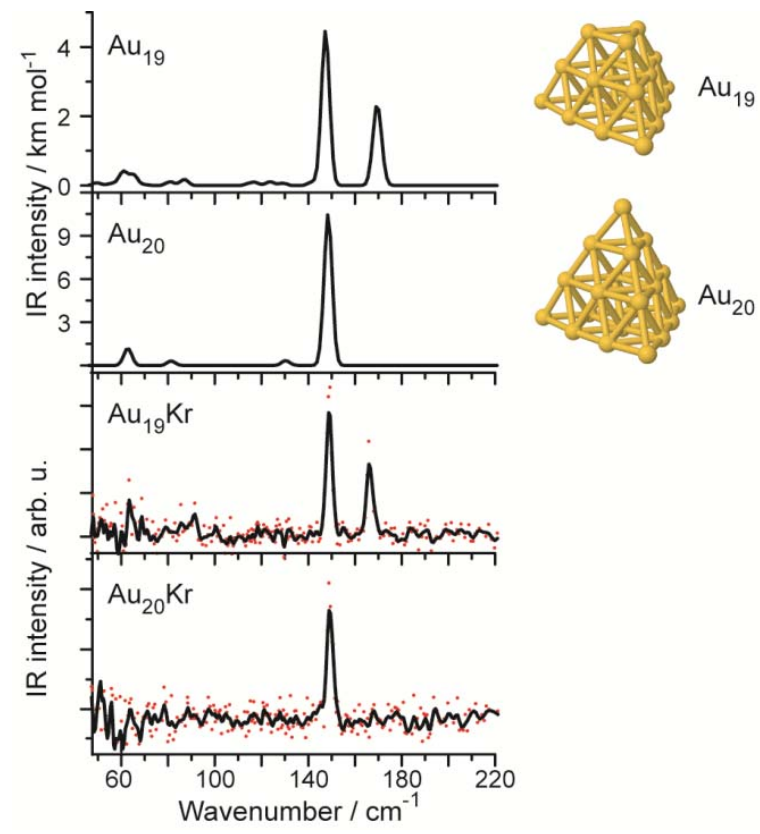

Figure 5: Experimental IR-MPD spectra of $\mathrm{Au}_{19} \mathrm{Kr}$ and $\mathrm{Au}_{20} \mathrm{Kr}$ and calculated far-IR spectra for $\mathrm{Au}_{19}$ and $\mathrm{Au}_{20}$. For details see Ref. [29]

The good agreement between the experimental IR-MPD spectra of $\mathrm{Au}_{19} \mathrm{Kr}$ and $\mathrm{Au}_{20} \mathrm{Kr}$ and the harmonic vibrational spectra of the bare clusters allows for the conclusion that in these cases i) the messenger is not significantly perturbing the vibrational properties of the gold clusters and ii) the vibrations are largely harmonic. This may not be generally true and, particularly for small clusters, larger deviations between the spectra and the predictions can be found.

The influence of $\mathrm{Kr}$ binding on the predicted harmonic IR spectra of $\mathrm{Au}_{7}$ is illustrated in the upper panels of Figure 6. Upon binding $\mathrm{Kr}$ the relative intensities of the vibrational modes change in a manner dependant on the location of the binding. Using dispersion-corrected DFT one finds similar binding energies for all the binding sites shown $\left(\Delta E_{\mathrm{B}} \approx 0.1 \mathrm{eV}\right)$. Nevertheless, at $100 \mathrm{~K}$ the binding to the plane is (for entropic reasons) significantly favoured as illustrated by the $80 \%$ isosurface for the presence of $\mathrm{Kr}$ as obtained from a molecular dynamics simulation (MD). The finite temperature IR spectrum of $\mathrm{Au}_{7} \mathrm{Kr}$ obtained from the autocorrelation function of the dipole moment in the MD simulations gives a good match to the experimental spectrum, but the comparison with a spectrum for the bare $\mathrm{Au}_{7}$ obtained in the same manner reveals only a minor influence of the $\mathrm{Kr}$ ligand. A closer analysis of the nuclear dynamics shows that this deviation from the linear absorption spectrum, and in particular the broadening of the highest frequency peak, is due to anharmonic behaviour. MD simulations at higher temperature illustrate that the comparably weak bond in the inner rhombus of $\mathrm{Au}_{7}$ (Figure 
6, dashed line in the $\mathrm{Au}_{7}$-structure) can break resulting in an isomerisation between two equivalent forms of this cluster making it rather fluxional [61]. Such dynamical behaviour appears to not be special for this size but important also for larger sizes e.g. $\mathrm{Au}_{13}$ [62].

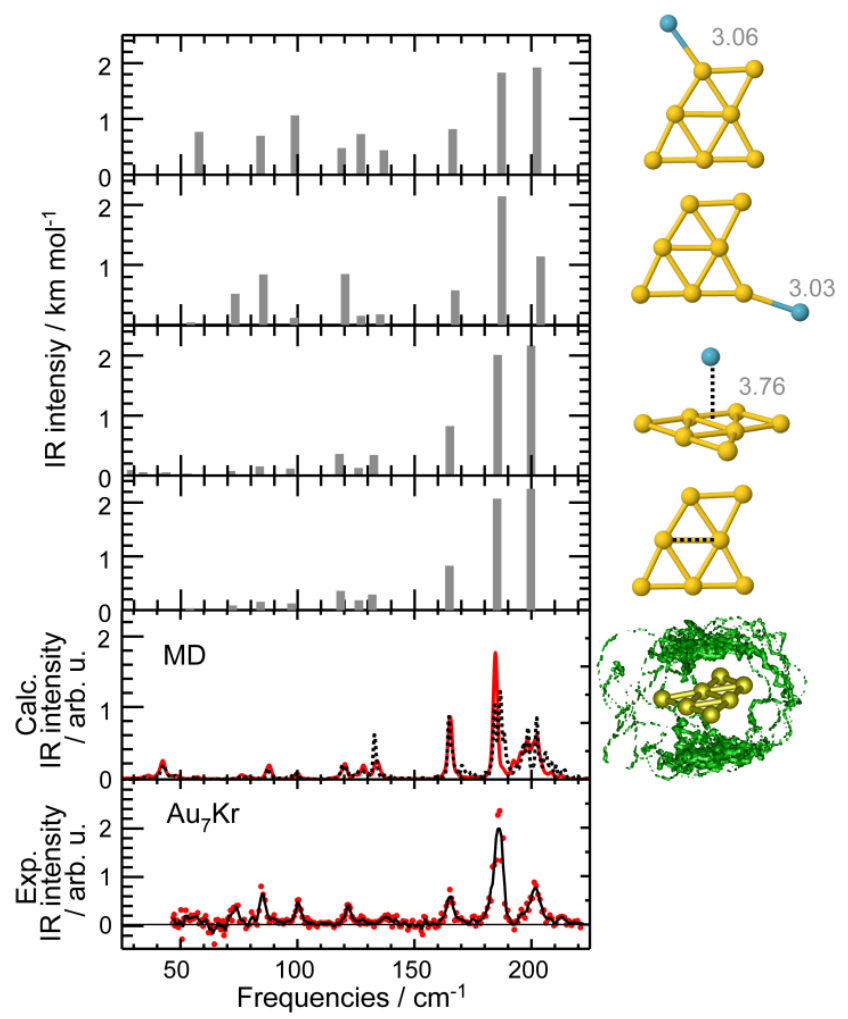

Figure 6: IR-MPD spectrum for $\mathrm{Au}_{7} \mathrm{Kr}$ (bottom panel), finite temperature IR spectra from molecular dynamics simulations at $100 \mathrm{~K}\left(2^{\text {nd }}\right.$ from bottom; red trace: $\mathrm{Au}_{7} \mathrm{Kr}$, dashed black: $\left.\mathrm{Au}_{7}\right)$ and harmonic IR spectra for the given limiting structures (remaining panels). At $100 \mathrm{~K}$ the $\mathrm{Kr}$ is not bound to a specific $\mathrm{Au}$ atom, but preferentially localized above and below the $\mathrm{Au}_{7}$-plane (green: $80 \%$ isosurface). From Ref. [61]

For still smaller clusters a similar treatment predicts a localized bonding of the $\mathrm{Kr}$ atoms in the cluster plane as illustrated in Figure 7. The $\mathrm{Kr}$ atoms are (at 100 $\mathrm{K})$ no longer mobile owing to two effects: Firstly the strength of the van-derWaals interaction scales with the number of $\mathrm{Au}$ atoms the $\mathrm{Kr}$ can interact with and thus the non-localised van-der-Waals bonding becomes weaker whilst the (localised) chemisorption in the plane becomes stronger. Further, due to the reduced size of the cluster the entropic favourability of the van-der-Waals bound $\mathrm{Kr}$ is reduced as there are fewer sites the $\mathrm{Kr}$ atom may sit. In these small $\mathrm{Au}_{n} \mathrm{Kr}_{m}$ complexes the $\mathrm{Kr}$ is relatively strongly bound by $0.15-0.20 \mathrm{eV}$ per $\mathrm{Kr}$ atom and thus 
the rare gas atoms need to be explicitly considered in order to explain the observed far-IR-MPD spectra. For all the sizes shown theory predicts the presence of isomers within about $0.02 \mathrm{eV}$, however in all cases only a single isomer (top row in Figure 7) is needed to satisfactorily explain the observed spectra [61].
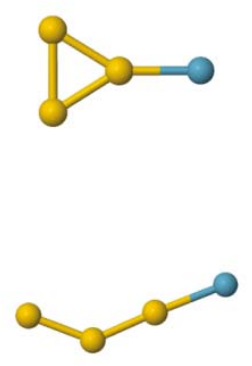

$\Delta \mathrm{E}=-0.02 \mathrm{eV}$
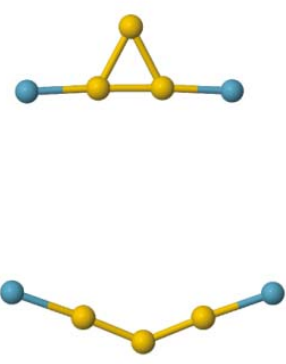

$\Delta \mathrm{E}=-0.02 \mathrm{eV}$
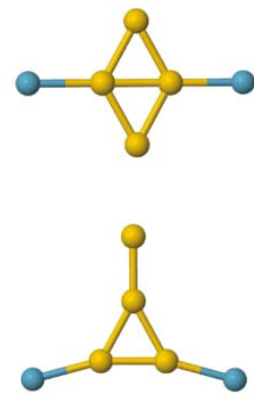

$\Delta \mathrm{E}=+0.01 \mathrm{eV}$

Figure 7: Structures of small $\mathrm{Au}_{n} \mathrm{Kr}_{m}$ clusters. The upper isomers are assigned by comparing IRMPD spectra with calculated finite temperature IR spectra whilst the lower row shows isomers close in energy (the difference is given relative to the upper row structure). The structures in the lower row may weekly contribute to the observed spectra or have an ionization energy too high to be detected [61]

\subsection{Doped Species}

In addition to studies on pure Au clusters, there has been a wealth of investigations, both experimental and theoretical, on gold clusters containing impurity atoms. By introducing such dopants new physical properties may emerge, e.g. related to modifications of the geometric and/or electronic structure. Depending on the dopant element it may act as an electron donor or acceptor in essence changing the number of valence electrons in the cluster [63-71]. Clearly such a topic has the potential to be massive in scope and here we only discuss the remarkable case of $\mathrm{Au}_{16}$ as being exemplary [72-75].

$\mathrm{Au}_{16}$ is found to form a stable cage as an anion. For the transition metal elements (including the isoelectronic $\mathrm{Cu}$ and $\mathrm{Ag}$ ) these dopant atoms can be incorporated inside this hollow $\mathrm{Au}_{16}{ }^{-}$cage with the s electrons of the metal being donated to the valence orbitals of the gold cluster. For $1 \mathrm{e}^{-}$donor species $(\mathrm{Cu}, \mathrm{Ni}$ and $\mathrm{Ag})$ this results in a stable anionic $18 \mathrm{e}^{-}$valence electron system with the slight further complication in the case of $\mathrm{Ni}$ arising due to the additional d-electrons [73]. For the other d-metals they instead donate 2 electrons into the valence electron system, i.e. the 18 valence electron system is realized in the neutral species, with the ani- 
onic clusters showing the opening of a new electronic shell. Notably, and of great interest for the development of future nano-materials, the resultant transition metal centres do not experience a quenching of the latent spin moments arising from the d-electrons in such an interaction. This provides the tantalizing possibility that the atomic-like magnetism can be preserved and protected by encapsulation into a golden cage [73].

Lastly, $\mathrm{Au}_{16}{ }^{-}$has been doped with the main group elements $\mathrm{Si}$, Ge and $\mathrm{Sn}$. With these elements an altogether different binding motif is observed. Instead of endohedral doping they displace a $\mathrm{Au}$ atom from the cage structure, in the latter two cases this atom is relocated to a capping site elsewhere on the gold cage whilst for $\mathrm{Si}$ this displaced atom becomes a "dangling" atom bound to the Si itself. This is in line with the recently observed strong Au-Si bonds and observation of gold based silane analogues $[65-67,75]$.

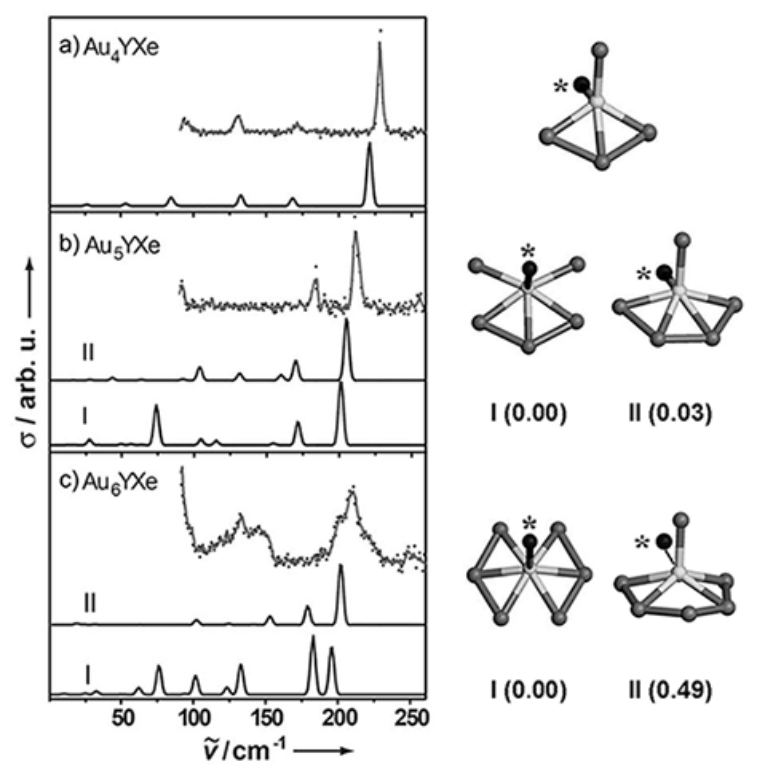

Figure 8: IR-MPD spectra and predictions of harmonic IR spectra for isomers of $A_{4} Y-A_{6} Y$ (light sphere: Y, dark spheres: Au, black sphere: $\mathrm{Xe}$ ). Relative energies are given in eV. From Ref. [76]

Comparable experimental information for the neutral species is scarce. To the best of our knowledge the only such data relates to the small yttrium doped gold clusters [76, 77] and a sample of the recorded IR-MPD spectra (measured by monitoring depletion of the parent xenon complexes) as well as comparisons to predicted harmonic spectra are presented in Figure 8. The agreement between theory and experiment for the $A_{4} \mathrm{Y}$ and $\mathrm{Au}_{5} \mathrm{Y}$ clusters is excellent and the well resolved and narrow (bandwidth limited) lines are accurately reproduced by the harmonic calculations. This is not so for $\mathrm{Au}_{6} \mathrm{Y}$ where the experimental spectrum consists of 
broad features which are not adequately reproduced in the harmonic prediction. This, it transpires, arises from an isomerization occurring on the time scale of the experiment between limiting forms of structure I passing through an intermediate of $\mathrm{D}_{6 h}$ symmetry. Interestingly, this intermediate structure is predicted to be a delocalised $\sigma$-aromatic system in the anionic cluster as it possesses $10 \mathrm{e}^{-}$(c.f. Hückel's $4 n+2$ electron rule). Further calculations reveal that such a cluster should possess an appreciable ring current as determined by calculating nucleus-independent chemical shifts, a hallmark of aromaticity [76].
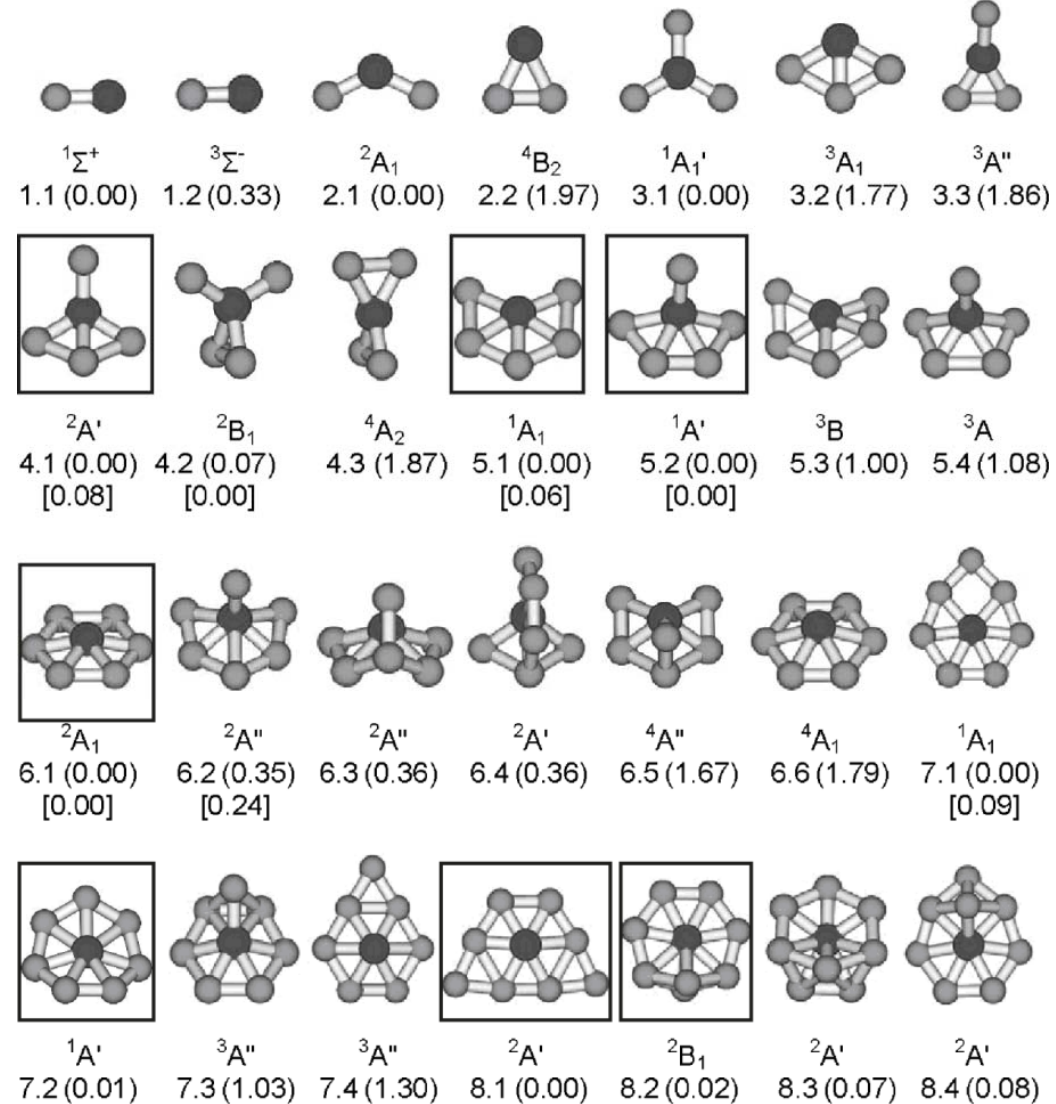

${ }^{3} \mathrm{~A} "$

${ }^{3} \mathrm{~A} "$

${ }^{2} \mathrm{~A}^{\prime}$

${ }^{2} B_{1}$

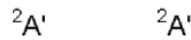

$7.2(0.01)$
$[0.00]$

$7.3(1.03) \quad 7.4(1.30)$

$8.1(0.00) \quad 8.2(0.02)$

$8.3(0.07) \quad 8.4(0.08)$
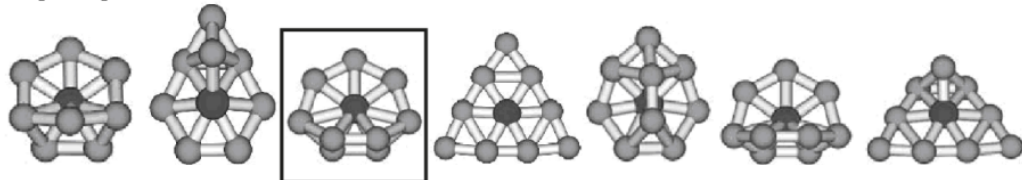

${ }^{4} \mathrm{~A}$

${ }^{4} \mathrm{~A} "$

${ }^{1} A_{1}{ }^{\prime}$

${ }^{1} A^{\prime}$

${ }^{3} A^{\prime \prime}$

${ }^{3} \mathrm{~A}^{1}$

$8.5(1.25) \quad 8.6(1.31) \quad 9.1(0.00)$

$\begin{array}{llll}9.2(0.15) & 9.3(0.15) & 9.4(1.00) & 9.5(1.38)\end{array}$ 
Figure 9: Predicted structures of $\mathrm{AuY}-\mathrm{Au}_{9} \mathrm{Y}$. The structures identified by comparison to experiment are framed. Relative energies are given for the BP86/cc-pVDZ-PP level of theory in eV (square brackets give values at the CCSD(T)/cc-pVTZ-PP level of theory). From Ref. [77]

All of the low energy isomers calculated for the $\mathrm{Au}_{n} \mathrm{Y}$ clusters are given in Figure 9 with the structures favoured on the basis of experiment and theory highlighted. In general the clusters are seen to adopt structures in which the number of $\mathrm{Au}-\mathrm{Y}$ bonds are maximized with the centred 7-member ring structure, which forms at $\mathrm{Au}_{7} \mathrm{Y}$, being a favoured motif for the larger clusters. This motif is not observed for pure gold clusters, but is stabilized by the incorporated $\mathrm{Y}$ due to it being a $10 \mathrm{e}^{-}$system (as a neutral subunit).

\section{Gold cluster complexes}

Knowledge of the bare gold cluster structures is a vital first step towards understanding the origins of their chemistry at the nano-scale. More direct insights into the function of the clusters in a reactive environment, however, can be obtained by studying complexes of these clusters with small ligands of catalytic relevance. From an experimental point of view the focus is often either on investigating the kinetics of complex formation (or transformation) or on structural (spectroscopic) characterization. To date complexes of gold clusters with a variety of ligands have been investigated but, in the interests of space, we limit the present discussion to two of the most studied: $\mathrm{O}_{2}$ and $\mathrm{CO}$. Additionally, the reaction kinetics have been the subject of two comparatively recent reviews [21, 78], which highlight the roles of gold and binary silver-gold clusters and their reactions with small molecules. As such in the present work we shall place the emphasis on the spectroscopic characterization of gold cluster complexes.

\subsection{Molecular oxygen}

Perhaps the most famous property of gold nano-particles is their ability to catalyze low-temperature oxidations using molecular oxygen as a feedstock [79-83]. These reactions are fascinating given that the typical mechanism for metal catalyzed oxidation reactions, the Mars-van-Krevelen mechanism [84], relies on the formation of oxide ions, $\mathrm{O}^{2-}$, a process which is known to be unfavourable for gold surfaces. Clearly, nano-scale materials may show a different chemistry and indeed small gold oxide clusters, i.e. species containing dissociated $\mathrm{O}_{2}$, can be produced [85-89]. Their formation, however, relies on the activation of molecular oxygen in the plasma plume formed during laser ablation, a highly energetic process quite 
unlike that found in a real catalyst. Nevertheless the question still remains: How does nano-scale gold react with and activate molecular oxygen?

\subsubsection{Anions}

The majority of the experimental investigations into gold cluster complexes with molecular oxygen have focused on the anions. Charged systems are experimentally easier to study, as previously mentioned, although the cations are generally less reactive towards oxygen with the exception of the decamer $\left(\mathrm{Au}_{10}{ }^{+}\right)[13]$.

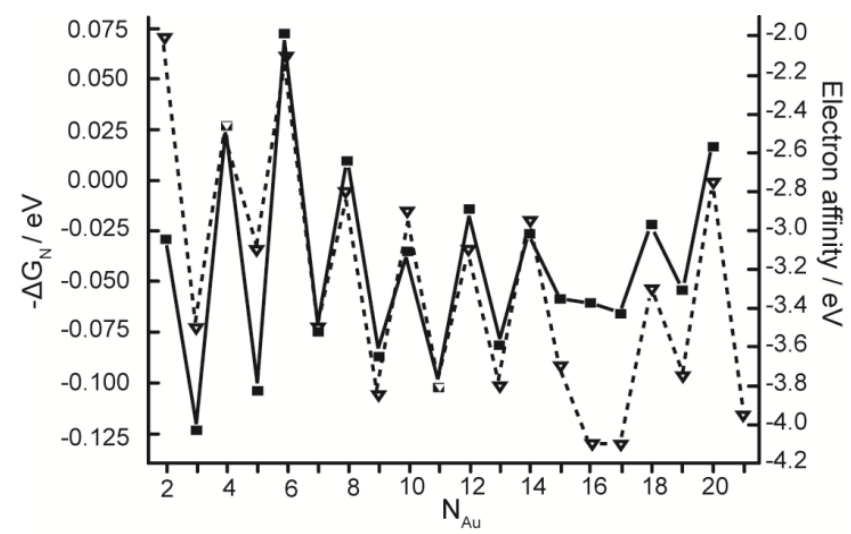

Figure 10 Free energy for the complex formation reaction $\mathrm{Au}_{n}{ }^{-}+\mathrm{O}_{2} \rightarrow \mathrm{Au}_{n} \mathrm{O}_{2}{ }^{-}$(closed squares, left axis) and the negative of the electron affinity of the corresponding neutral cluster (which is equal to the electron binding energy, $\mathrm{BE}$, in the anion; open triangles, right axis). Reprinted from Chem. Phys., Vol. 262, Salisbury B. E. et al. "Low temperature activation of molecular oxygen by gold clusters: a stoichiometric process correlated to electron affinity", 131, Copyright (2000) with permission from Elsevier

For the anions, only the even-.sized clusters, $\mathrm{Au}_{2 n}{ }^{-}$, show appreciable reactivity with $\mathrm{O}_{2}[13,90,91]$. This has been attributed to the alternating open/closed shell of the gold clusters arising from the $5 d^{10} 6 s^{1}$ electron configuration of the Au atom [90]. This alternating shell structure means the even sized anions (which have open shells) typically show lower electron binding energies (BE) than their neighbouring clusters. This oscillation in the $\mathrm{BE}$ of the clusters has been correlated with the energy for the association reaction $\mathrm{Au}_{n}{ }^{-}+\mathrm{O}_{2} \rightarrow \mathrm{Au}_{n} \mathrm{O}_{2}{ }^{-}$as shown in Figure 10. The only significant deviation from this correlation occurs at $\mathrm{Au}_{16}{ }^{-}$, which shows an anomalously high $\mathrm{BE}$ and no reaction with $\mathrm{O}_{2}$. This has been interpreted to the cage structure for $\mathrm{Au}_{16}{ }^{-}$which benefits from spherical aromaticity, stabilizing the anion [92].

This preferential reactivity of the even sized anions leads to a simple binding model for the complexes: The unpaired electron of the gold cluster is donated into the partially occupied HOMO of $\mathrm{O}_{2}$ (the MO diagram for which is shown in Fig- 
ure 11). Since this $\pi^{*}$ orbital is of $\mathrm{O}-\mathrm{O}$ anti-bonding character this weakens the oxygen bond to form a superoxo $\left(\mathrm{O}_{2}^{-}\right)$moiety (bond dissociation energy $398 \mathrm{vs}$. $499 \mathrm{~kJ} \mathrm{~mol}^{-1}$; see Figure 11). In this way the $\mathrm{O}_{2}$ becomes activated upon complexation with the gold cluster leaving it open to further attack.

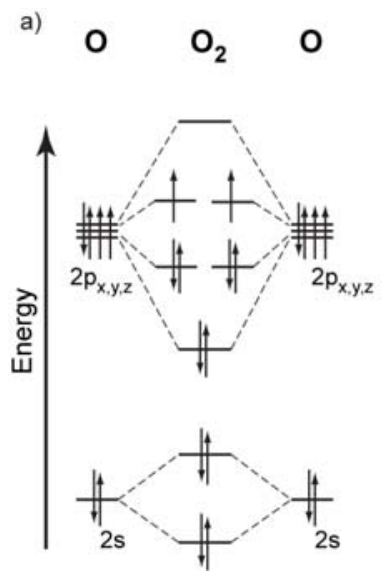

b)
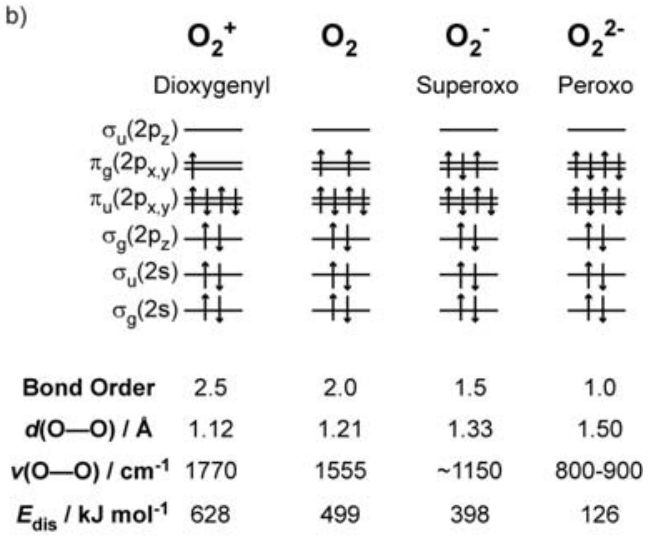

Figure 11: MO Scheme for $\mathrm{O}_{2}$ and the corresponding orbital occupancies for the different oxidation states of $\mathrm{O}_{2}$. Additionally the formal bond order, $\mathrm{O}-\mathrm{O}$ bond distance, vibrational frequency and bond dissociation energy are given for all the oxidation states. Numerical values from [93]

Evidence for this binding mechanism comes from multiple sources. Several Anion PES spectra have been recorded for these clusters and provide two crucial observations: Firstly, a vibrational progression is seen in the spectra of $\mathrm{Au}_{2} \mathrm{O}_{2}{ }^{-}$, $\mathrm{Au}_{4} \mathrm{O}_{2}^{-}$and $\mathrm{Au}_{6} \mathrm{O}_{2}^{-}$with stretching frequencies ranging between 1226 and 1443 $\mathrm{cm}^{-1}[14,15,94]$. Such a vibrational frequency corresponds to a partially activated $\mathrm{O}_{2}$, somewhere between physisorbed oxygen and a superoxo species. Secondly, when the experiment is performed at cryogenic temperatures it is possible to form the oxygen complexes with the odd-sized clusters. The resulting spectra, however, are almost identical to that of the parent bare gold anion, indicating that no electronic structure changes have occurred in the complexation, i.e. a purely van-derWaals interaction [15].

Another PES study [95], which focused on the larger anions $\left(\mathrm{Au}_{6}{ }^{-}-\mathrm{Au}_{20}{ }^{-}\right)$, found a transition in the binding geometry of the superoxo moiety from being bound to a single $\mathrm{Au}$ atom $\left(\mu^{1}\right)$ to two $\mathrm{Au}$ atoms $\left(\mu^{2}\right)$ with a critical size for the change at $\mathrm{Au}_{8}{ }^{-}$. This conclusion, however, is based on the supporting DFT calculations as no vibrational progressions were observed in the PES.

Direct evidence for the presence of superoxo species comes from IR-MPD spectroscopy. The IR-MPD spectra for the dioxygen complexes of the anionic species $\mathrm{Au}_{4}^{-}-\mathrm{Au}_{20}{ }^{-}$clearly show absorption between 1050 and $1100 \mathrm{~cm}^{-1}$ (Figure 12) [11]. This unambiguously demonstrates the presence of a superoxo moiety bound to the gold cluster as no other vibrational fundamentals are expected at 
these frequencies. The observed frequencies, however, disagree with the vibrational progressions recorded from Anion PES. This is most likely due to the nature of PES which is sensitive to both the anionic and the neutral states. The vibrational progressions in the Anion PES are now thought to instead arise from photodetachment to an electronically excited state of the neutral cluster which corresponds to the reaction of the gold cluster with singlet oxygen, rather than being representative of the superoxo state [15].

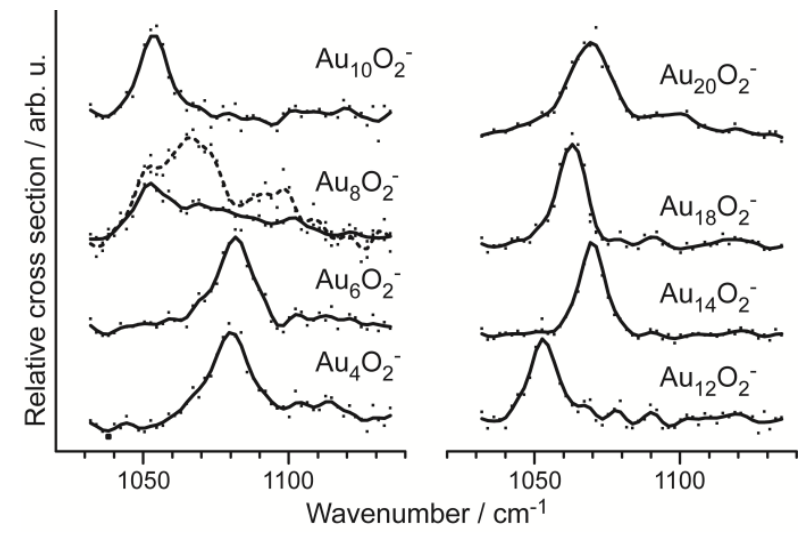

Figure 12: IR-MPD spectra for the even sized gold cluster complexes $\mathrm{Au}_{n} \mathrm{O}_{2}{ }^{-}$. All show an absorption in the energy range which corresponds to a superoxo stretch. Two traces are given for $\mathrm{Au}_{8} \mathrm{O}_{2}{ }^{-}$demonstrating the limiting cases of the spectra observed by varying the source conditions. From Ref. [11]

Two very different experiments, anion PES [95] and IR-MPD [11], permit an experimental cross validation for the special behaviour observed for the $\mathrm{Au}_{8} \mathrm{O}_{2}{ }^{-}$ complex. Both studies find that the observed experimental spectra are very sensitive on the exact source conditions employed. In the reproduced IR-MPD spectra these are given as two different traces for $\mathrm{Au}_{8} \mathrm{O}_{2}{ }^{-}$. Accompanying DFT calculations reveal that this is due to an adsorbate driven isomerisation between two forms of the $\mathrm{Au}_{8} \mathrm{O}_{2}{ }^{-}$cluster complex. Depending on the residence time in the reaction channel, either the cluster complex corresponding to the ground state $\mathrm{Au}_{8}$ structure (a 4-pointed star) or the excited state structure (an edge-capped centred hexagon) prevails. It transpires that the complex of the edge-capped centred hexagon structure is lower in energy than the 4-pointed star complex but a significant barrier to the rearrangement exists $(0.43 \mathrm{eV})$ hence the possibility for kinetic trapping [95]. Further, both investigations find that this structural change is accompanied with a transition from a $\mu^{1}$ bound complex for the 4-pointed star to a $\mu^{2}$ complex in the edge-capped centred hexagon. 


\subsubsection{Neutrals}

One of the suggested mechanisms for the catalytic activity of nano-dispersed gold particles builds upon the observation that gold clusters are found to become negatively charged when deposited over defect sites in oxide supports and, indeed, such systems have been shown to be catalytically active [1,2]. This, in part, drove the experimental and theoretical interest in the anionic gold cluster complexes with oxygen. Neutral clusters, however, are much more likely to be present in a real catalyst and thus it is interesting to investigate if and how neutral gold clusters interact with molecular oxygen.

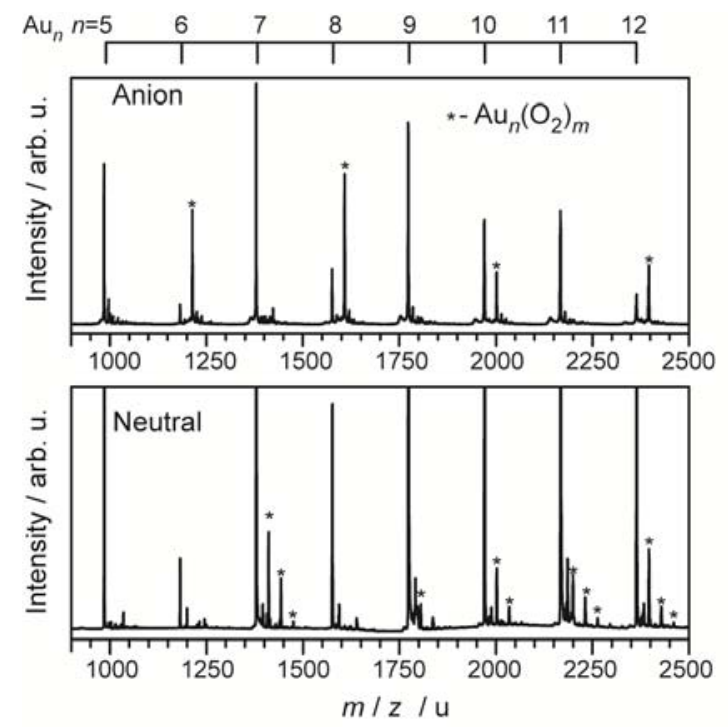

Figure 13: Mass spectra in the range of $\mathrm{Au}_{5}$ to $\mathrm{Au}_{12}$ when reacted with $\mathrm{O}_{2}$. The upper panel shows the anionic species (reacted at $-100{ }^{\circ} \mathrm{C}$ ), the lower the neutral (reacted at $-177^{\circ} \mathrm{C}$ ). All $\mathrm{Au}_{n}\left(\mathrm{O}_{2}\right)_{m}^{-/ 0}$ species are labelled with an asterisk. Other peaks in the mass spectrum relate to water and other impurities. Neutral clusters are ionized by $7.9 \mathrm{eV}$ photons from an $\mathrm{F}_{2}$ laser.

Figure 13 compares the mass spectra of the anionic gold cluster complexes and the neutral cluster complexes when using $7.9 \mathrm{eV}$ photons for ionization. As was mentioned earlier, the reactivity for the anions is dominated by the even-sized clusters whereas mass peaks corresponding to both even and odd-sized cluster complexes are observed in the neutral mass spectrum. The ionization energies (IEs) of gold clusters tend to be high and therefore the $7.9 \mathrm{eV}$ photons do not efficiently ionize all the clusters with a single photon. As such the ionization cross sections have a marked effect on the observed distribution. Unfortunately these cross sections are not known for the different species and so no definitive conclu- 
sions concerning the reactivity can be drawn from these mass spectra. Those species which can be ionized, however, can have spectroscopic probes applied to them. Figure 14 shows the IR-MPD spectra for such complexes of neutral gold clusters [12].

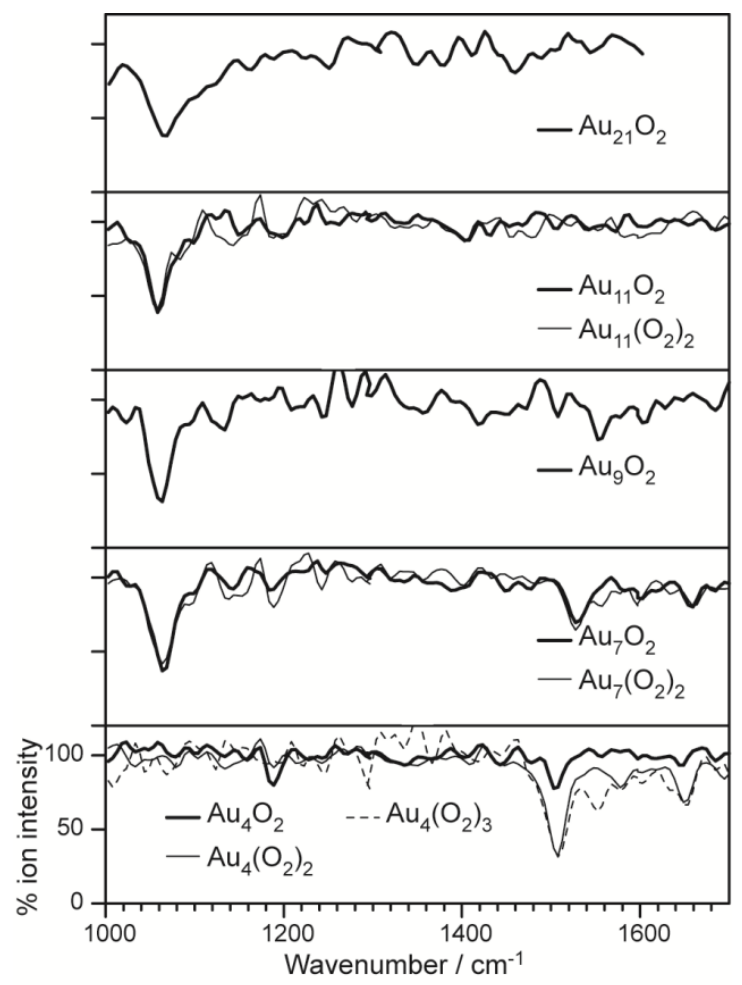

Figure 14: IR-MPD spectra for oxygen complexes of neutral gold clusters. The observed absorptions correspond to superoxo moieties $\left(\mathrm{O}_{2}^{-} ; \sim 1050 \mathrm{~cm}^{-1}\right)$ and physisorbed $\mathrm{O}_{2}\left(\mathrm{O}_{2}{ }^{0} ; \sim 1550 \mathrm{~cm}^{-1}\right)$. Reprinted with permission from: Woodham, A. P., et al. (2013) J. Am. Chem. Soc. 135: 17271730. Copyright 2013 American Chemical Society

For $\mathrm{Au}_{10}\left(\mathrm{O}_{2}\right)_{1-2}$ and $\mathrm{Au}_{12}\left(\mathrm{O}_{2}\right)_{1-3}$ no bands are observable in the range of 1000 $1700 \mathrm{~cm}^{-1}$. This indicates that either the oxygen dissociates upon binding to the gold clusters, resulting in the formation of a gold oxide which is not expected to possess a vibrational fundamental at these frequencies, or the oxygen is sufficiently weakly adsorbed to the cluster that the formally IR-inactive $\mathrm{O}-\mathrm{O}$ stretch is not sufficiently perturbed to become IR-active. As yet no evidence exists to distinguish the two scenarios.

The other complexes show vibration fundamentals in two ranges, one corresponding to a superoxo moiety between 1000 and $1100 \mathrm{~cm}^{-1}$, and is seen for all the odd-sized clusters. The second is due to a slightly activated $\mathrm{O}_{2}$ moiety, essentially 
physisorbed, at around $1500-1520 \mathrm{~cm}^{-1}$ and is observed for $\mathrm{Au}_{7}\left(\mathrm{O}_{2}\right)_{1-2}$ as a minor additional peak and as the only feature in $\mathrm{Au}_{4}\left(\mathrm{O}_{2}\right)_{1-3}$. The extreme similarity between the spectra with a single $\mathrm{O}_{2}$ and those with multiple $\mathrm{O}_{2}$ units suggests that these additional oxygen molecules are purely spectators and only bound by a weak van-der-Waals type interaction. The presence of superoxo vibrations for the oddsized clusters is in line with the binding model derived from the anionic clusters, where a single unpaired electron is required for donation into the $\pi^{*} \mathrm{HOMO}$ of the oxygen molecule. The smaller band indicating un-activated $\mathrm{O}_{2}$ in $\mathrm{Au}_{7}\left(\mathrm{O}_{2}\right)$ is most likely due to an isomeric structure, either in the binding position of the $\mathrm{O}_{2}$ or of the gold cluster core structure itself.

As with the anionic clusters, interpretation of the data is aided by supporting quantum chemical calculations. The suggested structures (Figure 15) are not the putative global minima, which are always found to be dissociated species [96100], but the lowest energy structures which contain a molecular $\mathrm{O}_{2}$. Of these structures the gold cluster cores for $\mathrm{Au}_{7} \mathrm{O}_{2}$ and $\mathrm{Au}_{9} \mathrm{O}_{2}$ do not have the known structures for the bare neutral clusters, but rather resemble the corresponding cationic clusters (also reproduced in Figure 15). This demonstrates yet again the importance of structural flexibility in these systems. Upon binding $\mathrm{O}_{2}$ the neutral gold cluster transfers an electron into the $\pi^{*} \mathrm{HOMO}$, activating the $\mathrm{O}_{2}$, and becoming formally positively charged. In response to this change in charge state the cluster then undergoes a structural rearrangement to its preferred geometry for this charge state. For the tetramer, no such rearrangement is observed as the gold cluster core remains neutral upon binding the $\mathrm{O}_{2}$.

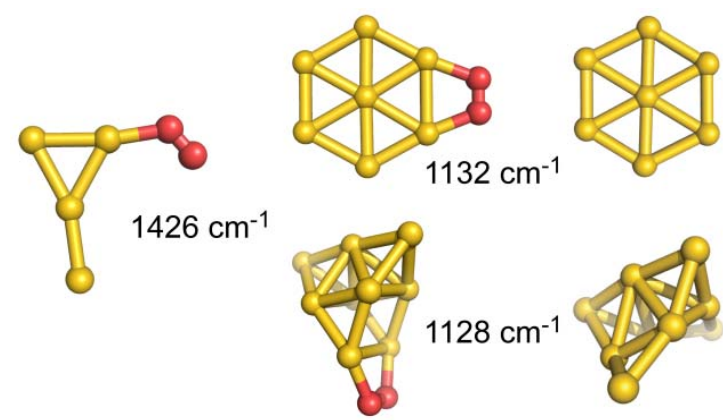

Figure 15: Structures of $\mathrm{Au}_{4} \mathrm{O}_{2}, \mathrm{Au}_{7} \mathrm{O}_{2}$ and $\mathrm{Au}_{9} \mathrm{O}_{2}$. The theoretically predicted vibrational frequency for the $\mathrm{O}-\mathrm{O}$ stretch is given for the complexes. For comparison the bare cationic cluster structures of $\mathrm{Au}_{7}^{+}$and $\mathrm{Au}_{9}^{+}$are also presented. There is a clear similarity between the neutral cluster complex and the cationic bare cluster

Given that the reactivity is dependent upon the IE of the cluster one can speculate as to the absence of the complexes $\mathrm{Au}_{13} \mathrm{O}_{2}$ to $\mathrm{Au}_{19} \mathrm{O}_{2}$. These clusters all possess an IE similar to, or less than, that of $\mathrm{Au}_{7}$, however, given that these clusters are metallic the resultant positive charge will become increasingly diluted throughout the cluster core as it increases in size. This charge dilution will result 
in a weaker ion-ion interaction with the formed superoxo moiety and thus the complexation will become thermodynamically less favourable. At $\mathrm{Au}_{21}$ the magic shell closing of a $20 \mathrm{e}^{-}$system dramatically lowers the IE, making the complex formation favourable once again.

While the differences in the reactivity pattern of neutral and anionic gold clusters are essentially due to the cluster's charge, more subtle variations in charge density can be realized by electron donating or accepting ligands. One example of how the reactivity can be modified in this way is the activity of partially hydrogen covered cationic gold clusters towards oxygen. As has been previously mentioned, the gold cations are thought to be unreactive towards molecular oxygen with the exception of $\mathrm{Au}_{10}{ }^{+}$[13]. Upon binding $\mathrm{H}_{2}$, however, all of the even sized clusters $\left(\mathrm{Au}_{2}{ }^{+}, \mathrm{Au}_{4}{ }^{+}\right.$and $\left.\mathrm{Au}_{6}^{+}\right)$are found to now bind one $\mathrm{O}_{2}$ molecule [101]. This cooperative effect is based on molecular hydrogen ligands acting effectively as electron donors, i.e. increasing electron density at the gold, and thereby enabling the activation of $\mathrm{O}_{2}$ via single electron transfer as discussed above.

These combined investigations reveal that oxygen activation, at least in the gas phase, is dependent upon the ability of the gold cluster to transfer an electron into the $\pi^{*}$ HOMO of the oxygen molecule. Thus only certain clusters are able to activate oxygen upon complexation. The extent of activation is comparable for all sizes and charge states, as the observed vibrational frequencies are similar for all the species presented. Lastly, the dynamic nature of the system is often very important for the observed reactivity, with the neutral clusters undergoing, sometimes dramatic, rearrangements in response to oxygen adsorption.

\subsection{Carbon Monoxide}

The prototypical reaction for modelling oxidative reactions with gold nano particles is the oxidation of carbon monoxide. Historically this reaction is of great significance as it was the first one observed to be catalyzed by gold nano-particles and spawned the entire research field [79, 80]. The reaction serves as a useful model for other oxidative reactions as well as being of industrial interest in its own right. For example with the possibilities to selectively remove $\mathrm{CO}$ from process gases (e.g. $\mathrm{CO}$ from $\mathrm{H}_{2}$ gas) or use as a scrubbing agent in water based fuel cells or car catalytic converters.

The binding of carbon monoxide to transition metals is a well-known phenomenon, typically described by the Blyholder model [102]. The Blyholder model assumes a two component bonding interaction; one component is electron donation from the CO HOMO, which is of $\sigma$-bonding character, into an empty, correctly symmetry adapted orbital of the metal centre. This occurs concurrently with backdonation from filled metallic d-orbitals into the empty $\pi^{*}$ LUMO of the CO (the second component). From such a binding model one can clearly see that metal$\mathrm{CO}$ binding will be favoured for metallic centres which contain a partially filled 
set of d-orbitals. For gold clusters this is not the case as the d-orbitals are completely filled and this is reflected in the weak binding of CO to gold surfaces [103] and indeed the rarity of gold-carbonyl complexes in both the solid and solution phases.

Similar to molecular oxygen the binding to the metal centre involves orbitals which are of $\mathrm{C}-\mathrm{O}$ anti-bonding character and so IR spectroscopy serves as an excellent probe to the local coordination environment of the $\mathrm{CO}$ molecules, and changes in the $\mathrm{C}-\mathrm{O}$ stretching frequency are routinely used to identify binding sites, motifs and strengths, and in particular the oxidation state of the metal, both on surfaces as well as in cluster carbonyls [104-106].

The properties of charged gas-phase clusters (both cations and anions) and their complexes with $\mathrm{CO}$ have been investigated in two regimes, a low pressure one and a high pressure one. In the high pressure regime the binding is best described by the Lindemann model for association reactions. Under such a model an initially formed encounter complex $\left(A B^{*}\right)$ is in equilibrium with the separated reagent species $(A$ and $B)$ and requires collision with a third body $(M)$ to undergo collisional cooling to form the product species $(A B)$ and an excited third body $\left(M^{*}\right)$ as shown in Equation (2). Conversely, conditions where such three body collisions are effectively absent with other cooling processes dominating (i.e. radiative cooling or uni-molecular decay) is defined as the low pressure regime.

$$
A+B \Leftrightarrow A B^{*}+M \rightarrow A B+M^{*}
$$

For cationic and anionic clusters the behaviour in the low pressure regime is similar [91, 107-109]. The interactions between the gold clusters and carbon monoxide are determined by simple electrostatic considerations and the ability of the resultant complexes to deposit the adsorption enthalpy into vibrational degrees of freedom. The first of these effects weakens the enthalpy of binding as the charge becomes diluted throughout the metallic droplet of the cluster with the binding enthalpy rapidly approaching that of the single crystal surface [109]. For $\mathrm{Au}_{n}{ }^{+}$clusters the binding energy has been experimentally determined to decrease for increasing cluster size from about $1 \mathrm{eV}$ for $n=5$ to below $0.65 \mathrm{eV}$ for $n>26$ and is calculated to be as high as $1.5 \mathrm{eV}$ for the cationic dimer $[109,110]$. As the binding energy is conserved in the complex the smallest clusters are prone to fragmentation upon $\mathrm{CO}$ binding such that they are often difficult to characterize experimentally. For example $\mathrm{Au}_{3} \mathrm{CO}^{+}$has been seen to form from initially formed $\mathrm{Au}_{4} \mathrm{CO}^{+}$ and subsequent elimination of a neutral Au atom [109].

In the high pressure regime, where collisional de-excitation dominates, the clusters can form multiply coordinated complexes, with saturation limited compositions being observed. Example mass spectra for the ionic and neutral species are reproduced in Figure 16. The saturation compositions appear not to be determined by simple electron counting rules [111,112], from which one would predict the presence of many $\mathrm{Au}_{n}(\mathrm{CO})_{m-1}{ }^{-}$compositions when comparing between the cationic and anionic species (the change from cation to anion involves the addition of $2 \mathrm{e}^{-}$, cor- 
responding to one $\mathrm{CO}$ ligand). Instead it is found that for the cationic clusters and the smaller $(n<6)$ anionic clusters the number of low coordination gold atoms ( 4 or fewer gold bonding partners) seems to provide a 1:1 correspondence with the $\mathrm{CO}$ saturation limit (for the gold cluster structures see Figure 2). In addition to this several cluster sizes exhibit metastable compositions which convert to a higher saturation coverage number with increased CO concentrations, the data are summarized in Table 1. The metastable compositions are attributed to adsorption driven structural rearrangements, again demonstrating the importance of structural flexibility in the chemistry of gold clusters $[111,113]$. Similar adsorption driven structural changes have also been observed with Anion PES [114-117]. The structural rearrangements can be quite dramatic and attempt to maximize the number of low coordination number atoms to which the $\mathrm{CO}$ can bind (For example see Figure 17). In some cases these structural transformations are observed to occur even with the addition of a single $\mathrm{CO}$ molecule.

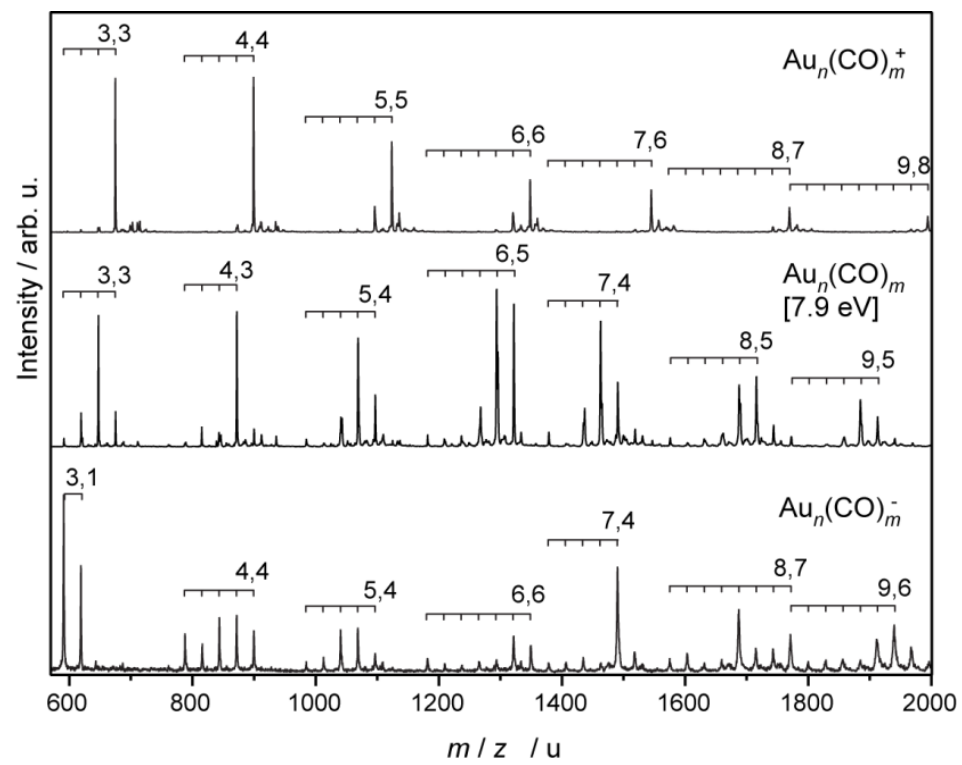

Figure 16: Mass spectra for cationic (top), neutral (middle) and anionic (bottom) gold cluster carbonyl complexes. The compositions are given as $(n, m)$ labels for the saturated species. The neutral mass spectrum was recorded after ionization with $7.9 \mathrm{eV}$ photons 


\begin{tabular}{|c|c|c|c|}
\hline Cluster & Anion $^{\#}$ & Neutral $^{\S}$ & Cation \\
\hline 3 & $\geq 1$ & $3(3)$ & 3 \\
\hline 4 & 4 & $3(3)$ & 4 \\
\hline 5 & $4(4)$ & $4(5)$ & 4,5 \\
\hline 6 & $6(4)$ & 5 & 5,6 \\
\hline 7 & $4,5(4)$ & $4(6)$ & 6 \\
\hline 8 & $4,7(5)$ & $5(7)$ & 7 \\
\hline 9 & $6(6)$ & $5(7)$ & 8 \\
\hline
\end{tabular}

Table 1: CO saturation number for different gold cluster sizes. Where two values are given this indicates evidence for a meta-stable intermediate saturation, which is observed until higher $\mathrm{CO}$ pressure. For neutral clusters saturation may not be fully reached for the mass spectrum shown in Figure 14. \# values in parentheses from Ref.[118]. § values in parentheses from Ref.[119]

Anion PES also provides further evidence for $\mathrm{CO}$ saturation effects for $\mathrm{Au}_{2}{ }^{-}-$ $\mathrm{Au}_{5}{ }^{-}$. Here successive addition of $\mathrm{CO}$ is accompanied by a marked red-shift in the observed EA of the cluster, owing to the $\sigma$-donation from the $\mathrm{CO}$ which destabilizes the HOMO of the gold cluster. After reaching saturation, however, each successive $\mathrm{CO}$ molecule induces a minor blue-shift owing to the formation of a solvation shell, which again stabilizes the charged species. In this way saturation compositions of $\mathrm{Au}_{2}(\mathrm{CO})_{2}{ }^{-}, \mathrm{Au}_{3}(\mathrm{CO})_{2}{ }^{-}, \mathrm{Au}_{4}(\mathrm{CO})_{3}{ }^{-}$and $\mathrm{Au}_{5}(\mathrm{CO})_{4}{ }^{-}$were determined $[116,117]$.
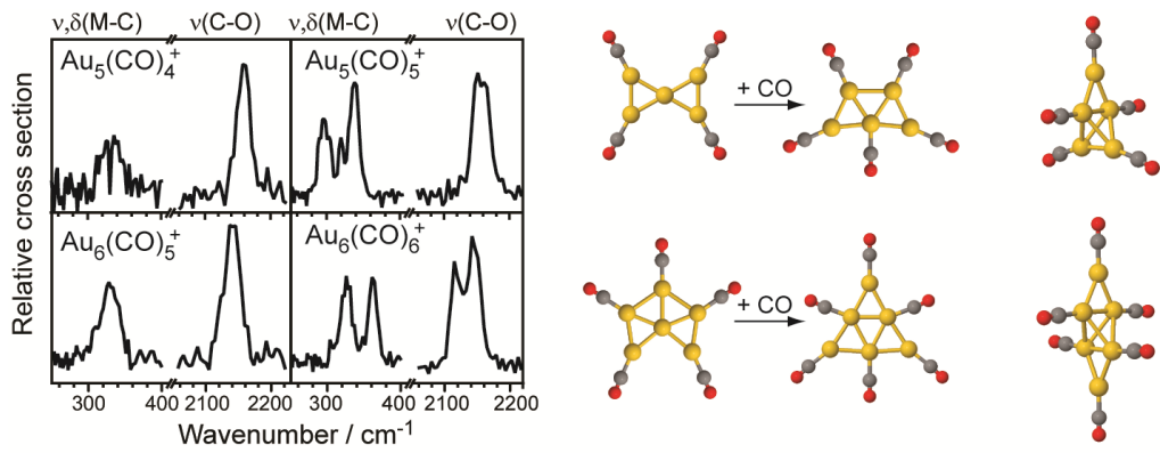

Figure $17 \mathrm{IR}$ spectra of $\mathrm{Au}_{5}(\mathrm{CO})_{4,5}{ }^{+}$and $\mathrm{Au}_{6}(\mathrm{CO})_{5,6}{ }^{+}$. For the lower coverage intermediate saturation is observed, leading to the suggestion of a structural rearrangement upon addition of the next $\mathrm{CO}$ molecule. Initially a transformation between $2 \mathrm{D}$ isomeric structures has been suggested [111] . More recent DFT calculations predict the formation of 3D structures upon CO addition to the planar bare gold clusters (right most structures) [113].

A summary of the observed $\mathrm{CO}$ stretching frequencies as a function of cluster charge, size and coverage as determined via IR-MPD spectroscopy is presented in Figure 18. For the smallest cationic clusters the observed $v(\mathrm{CO})$ values are blue shifted relative to that of the free $\mathrm{CO}\left(2143 \mathrm{~cm}^{-1}\right)$. This shows that the binding of 
$\mathrm{CO}$ to gold clusters is non-classical as electrostatic effects dominate over the $\pi$ back-donation [120]. As the cluster size increases, however, the shift from the free $\mathrm{CO}$ value reduces. This can be interpreted as the clusters functioning as metallic droplets and the charge becoming increasingly diluted throughout the structure. The effect of such a dilution can be modelled [121] and the expected shift is 15 $\mathrm{cm}^{-1}$ over the range $\mathrm{Au}_{3}{ }^{+}-\mathrm{Au}_{10}{ }^{+}$. The actual, experimentally observed, shift is on the order of $40 \mathrm{~cm}^{-1}$ indicating that some $\pi$-back-donation may play a role in the binding of $\mathrm{CO}$, particularly for the larger clusters [111]. The anionic clusters behave in a manner which approximately mirrors that of the cations. The $\mathrm{CO}$ stretching frequencies are uniformly red-shifted compared to the free $\mathrm{CO}$ value as the extra electron density of the anion makes $\pi$-back-donation more favourable, such that it dominates the electrostatic effect. As the clusters increase in size this extra density again becomes diluted throughout all metal atoms, lessening the effect and giving rise to the observed increase in $v(\mathrm{CO})$ as the cluster size increases. As with the cations, the contribution to this shift from purely electrostatic considerations should be $15 \mathrm{~cm}^{-1}$ over the series $\mathrm{Au}_{3}{ }^{-}-\mathrm{Au}_{10}{ }^{-}$whilst the observed shift is $80 \mathrm{~cm}^{-1}$ again likely due to the role of $\pi$-back-donation and other bonding effects [112]. With increasing CO coverage there is also a blue shift in the observed $v(\mathrm{CO})$ of $\sim 4$ $\mathrm{cm}^{-1}$ per $\mathrm{CO}$ molecule which arises due to increased competition for the electron density of the anionic cluster, reducing the share of this density each carbonyl receives. Perhaps expectedly, both the anions and cations tend towards a common value with increasing cluster size, which corresponds to $v(\mathrm{CO})$ found for neutral gold carbonyls (see below).

In terms of $v(\mathrm{CO})$, the binding of a single $\mathrm{CO}$ molecule is found to be largely insensitive to open/closed shell oscillations in the charged clusters, in contrast to $\mathrm{O}_{2}$ (see before) or NO [122]. For the CO saturated charged clusters some oscillatory behaviour becomes evident (Figure 18), with the even sized, i.e. open-shell, species exhibiting the lower $v(\mathrm{CO})$. It is not clear however, if this slightly stronger activation of the $\mathrm{CO}$ ligands is entirely due to the electronic structure or if geometric effects and a change in $\mathrm{CO}$ coverage also contribute. 


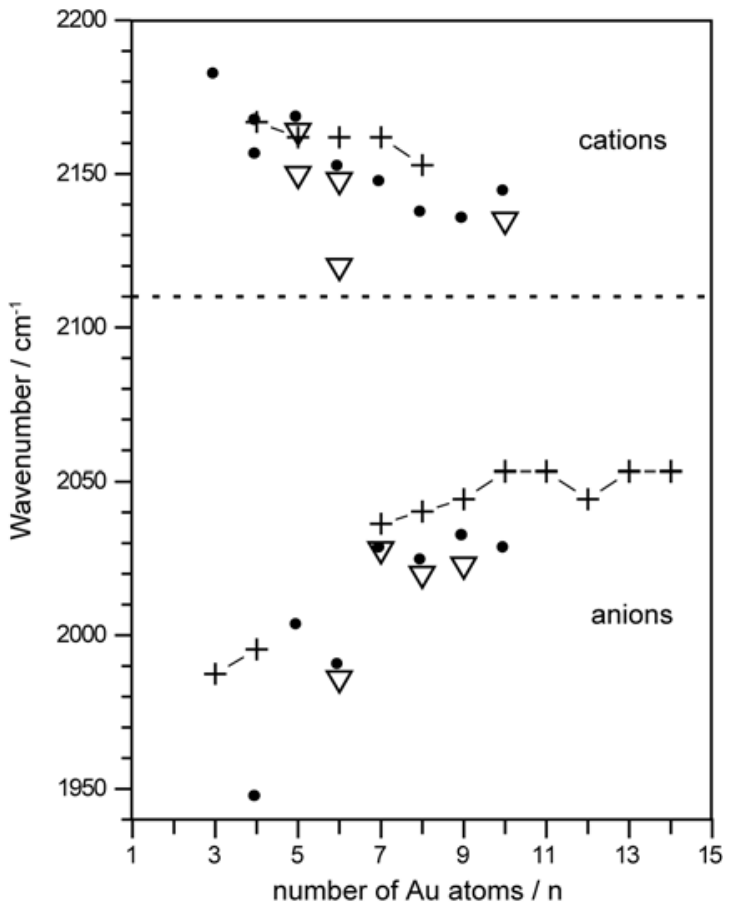

Figure 18: Summary of the observed $v(\mathrm{CO})$ values for anionic and cationic gold cluster carbonyls. The values for the monocarbonyl complexes are marked by the connected crosses whilst the saturated ones by closed circles. In the cases where intermediate saturation complexes are formed the high $\mathrm{CO}$ pressure compositions are represented by open triangles. The dashed line indicates the $v(\mathrm{CO})$ value for $\mathrm{CO}$ adsorbed on gold surfaces. Reprinted with permission from: Fielicke, A., et al. (2005) J. Phys. Chem. B 109: 23935-23940. Copyright 2005 American Chemical Society.

Lastly, saturation composition data for the neutral cluster complexes are also shown in Figure 16. The observed compositions when ionizing with $7.9 \mathrm{eV}$ photons are almost identical when an $\operatorname{ArF}$ laser $(6.4 \mathrm{eV})$ is used for photoionization [119] indicating that binding of CO significantly lowers the IE, as the bare gold clusters have IEs in the range of about 7-9 eV [123]. In general, the neutral complexes are observed to bind fewer carbonyl ligands compared with the anionic and cationic cluster complexes. This may be due to the lack of an ion - dipole interaction which plays a significant part in the energetics for the charged species as the $\sigma$-donation and $\pi$-back-donation are both relatively weak for these gold cluster carbonyls (as seen before). For the larger clusters $\left(\mathrm{Au}_{9}-\mathrm{Au}_{68}\right)$ reactivity data in the low-pressure regime also exists [124]. These possess a behaviour similar to the charged species, in that the reactivity is dependent upon the ability of the cluster to stabilize the energy of adsorption within the molecular degrees of freedom. In addition to this, an approximate odd-even oscillation is observed, with the even clusters demonstrating enhanced reactivity. This has been explained as due to the $2 \mathrm{e}^{-}$ 
donor nature of $\mathrm{CO}$ resulting in favourable binding to the (closed shell) even sized clusters, presumably as the LUMO orbital is readily able to accommodate the two electrons of $\mathrm{CO}$. The absence of a similar behaviour in the charged clusters would then be attributed to the ion-dipole interaction being the dominate term in the binding. Indeed, odd-even oscillations in $v(\mathrm{CO})$ are observed for partially $\mathrm{CO}$ saturated neutral gold clusters, with the open-shell systems showing lower $v(\mathrm{CO})$, as in the case of the charged clusters [125]. The absolute values are between 2070$2100 \mathrm{~cm}^{-1}$, i.e. just half-way between the $v(\mathrm{CO})$ frequencies of the $\mathrm{CO}$ saturated cations and anions, as predicted by the charge dilution model [121].

This survey of the carbonyl complexes with gold in all three charge states reveals that their interaction is atypical when compared with other transition metals. The effect of $\pi$-back-bonding is minimal owing to the stability of the filled $5 \mathrm{~d}$ orbitals and so the trends in the $\mathrm{CO}$ bond strength are instead dominated by electrostatic effects and the $\sigma$-donation from the carbonyl into the LUMO of the cluster. The saturation compositions of the gold clusters appear to be determined not by electron counting rules, as with other transition metal carbonyls, but rather from the interplay of electronic and structural considerations, with the availability of low-coordinate gold atoms being of paramount importance. This requirement for low-coordinate gold atoms is seen to be the driving force for large structural changes upon complexation with $\mathrm{CO}$ in order to maximize the number of available binding sites.

\subsection{Carbon Monoxide and Oxygen}

To gain further mechanistic insight into oxidation reactions on gold nanoparticles co-adsorbate species, e.g. clusters containing both $\mathrm{O}_{2}$ and $\mathrm{CO}$, have been studied. Whilst studying the gold cluster complexes with a single kind of ligand allows for analysis of their individual binding mechanisms, it is their interaction which determines the chemistry in the co-adsorbate species. Under conditions where two different reactants are present two scenarios are possible: the two species may compete with each other for binding sites and the resultant cluster distribution will reflect the ratio of the two sticking probabilities. Alternatively the presence of one ligand may change the probability of the other ligand to bind, e.g. enhance it. One example for such cooperative binding of $\mathrm{H}_{2}$ and $\mathrm{O}_{2}$ on cationic gold clusters has been discussed before, and for other ligands similar effects have been reported [101, 126-128].

Cooperative effects also determine the binding of $\mathrm{CO}$ and $\mathrm{O}_{2}$ to the even-sized gold cluster anions. The preadsorption of either $\mathrm{CO}$ or $\mathrm{O}_{2}$ can lead to massive enhancements of the reactivity for the next ligand with $\mathrm{Au}_{4}{ }^{-}$showing an enhancement of 18:1 [126]. Such behaviour again fits into the frontier orbital pictures which have been discussed before. In the case of initial $\mathrm{O}_{2}$ binding the resultant gold cluster core will be similar to the corresponding neutral cluster as it has trans- 
ferred its excess electron density into the $\mathrm{O}_{2}$, thus there is a vacant orbital which can accept two electrons from $\mathrm{CO}$. Alternatively, if the $\mathrm{CO}$ complex forms first then the donation of two electrons pushes the SOMO up in energy making its subsequent donation into an $\mathrm{O}_{2}$ more favourable. Indeed a plot of the reaction probabilities as a function of the number of electrons in the cluster (Figure 19) shows a remarkable similarity between the activity of the cluster carbonyl and the bare cluster with 2 additional gold atoms (i.e. 2 extra valence electrons).

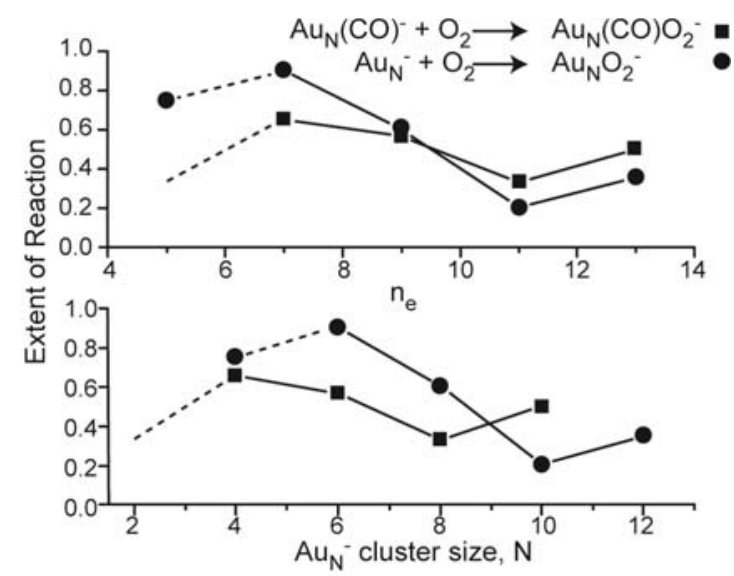

Figure 19: Comparison of reaction extents for the association reactions of $\mathrm{Au}_{n} \mathrm{CO}^{-}$(closed squares) and $\mathrm{Au}_{n}{ }^{-}$(closed circles) with $\mathrm{O}_{2}$. The top panel shows these as a function of valence electron count, whilst in the bottom panel as a function of cluster size. Reproduced with permission from: Wallace, W. T. and Whetten, R. L. (2002) J. Am. Chem. Soc. 124: 7499-7505. Copyright 2002 American Chemical Society.

With only one exception, this cooperative binding was not observed to change the rules for $\mathrm{O}_{2}$ adsorption in that only a single $\mathrm{O}_{2}$ was found to adsorb and only on the even sized gold anions. The exception being $\mathrm{Au}_{3}{ }^{-}$which, when studied at cryogenic temperatures, is seen to form the species $\mathrm{Au}_{3} \mathrm{COO}_{2}{ }^{-}$and $\mathrm{Au}_{3} \mathrm{CO}\left(\mathrm{O}_{2}\right)_{2}{ }^{-}$ [128]. Unfortunately it is not clear if this is an electronic structure effect induced by the $\mathrm{CO}$ binding or a physisorption of the $\mathrm{O}_{2}$ to the larger dipole of the $\mathrm{Au}_{3} \mathrm{CO}^{-}$ complex.

In addition to the $\mathrm{CO}$ and $\mathrm{O}_{2}$ co-adsorbates of these anionic clusters mass peaks which correspond to ions containing an odd number of $\mathrm{O}$ atoms have been observed $[17,126]$. Such species may be formed upon transferring one of the $\mathrm{O}$ atoms supplied by $\mathrm{O}_{2}$ to $\mathrm{CO}$, i.e. resulting in its oxidation, and release of $\mathrm{CO}_{2}$. For $\mathrm{Au}_{2}{ }^{-}$the reaction kinetics have been studied both experimentally and theoretically in great detail $[17,128,129]$ providing the realization of a catalytic cycle for $\mathrm{CO}$ oxidation (Figure 20). The cycle begins with formation of the oxygen complex which then reacts with a single $\mathrm{CO}$ molecule. Two possible structures of this intermediate are discussed, either a carbonate or a peroxyformate species. For the 
carbonate species of $\mathrm{Au}_{2}{ }^{-}$this then undergoes an Eley-Rideal reaction step with a second $\mathrm{CO}$ to evolve two $\mathrm{CO}_{2}$ molecules and regenerate the starting $\mathrm{Au}_{2}^{-}$. The alternative peroxyformate species also undergoes an Eley-Rideal reaction step to form a stabilized complex which then fragments to regenerate the bare gold cluster and evolve two $\mathrm{CO}_{2}$ molecules. A similar mechanism has been suggested for neutral $\mathrm{Au}_{2}$ but involving $\mathrm{Au}_{2}(\mathrm{CO})_{2}$ as a catalytically active species [62].

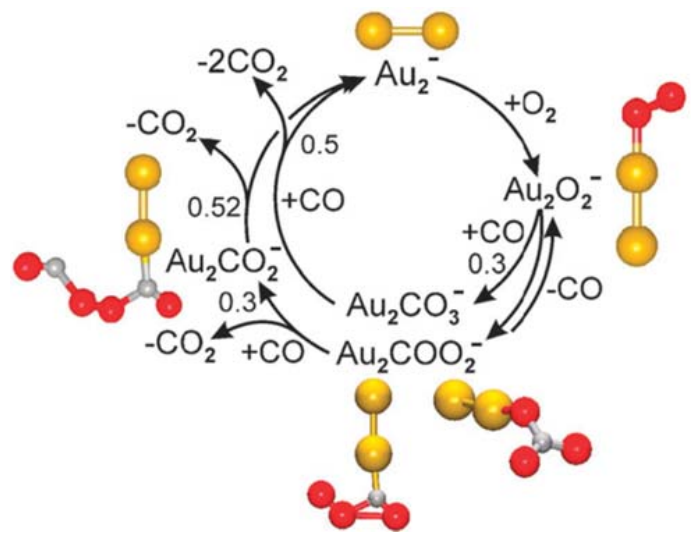

Figure 20: Mechanism for the catalytic oxidation of $\mathrm{CO}$ by $\mathrm{O}_{2}$ at $\mathrm{Au}_{2}{ }_{2}$. Reprinted from Appl. Catal. A, 291, Bernhardt, T. M. et al. "Size and composition dependence in CO oxidation reaction on small free gold, silver, and binary silver-gold cluster anions", Pages 170-178, Copyright 2005. With permission from Elsevier

The reactions between the neutral carbonyl clusters and $\mathrm{O}_{2}$ have also been investigated under multiple collision conditions using a fast flow reactor [119]. The reaction is monitored by observing the loss of carbonyl-complex signal when oxygen is present in the reaction cell. In order to exclude the effects of losses from scattering the observed depletion is compared with and normalized to the reduction in signal for a reaction cell filled with a partial pressure of $\mathrm{N}_{2}$, which is assumed to be unreactive and have a scattering cross section similar to that of $\mathrm{O}_{2}$. As with other studies of the neutral clusters the data is hampered by the high IEs of the gold clusters and as such only the odd sized clusters are measurable. For these species all of the observed carbonyl complexes react with $\mathrm{O}_{2}$ at $300 \mathrm{~K}$ with the cluster complexes $\mathrm{Au}_{3}(\mathrm{CO})_{2}$ and $\mathrm{Au}_{5}(\mathrm{CO})_{4}$ being local maxima in the reactivity which reduces at the largest cluster sizes investigated $\left(\mathrm{Au}_{9}(\mathrm{CO})_{6}\right)$. Unfortunately there is no clear electronic or structural argument to rationalize this observed variation in reactivity. 


\section{Conclusions}

This chapter has presented a comprehensive review of the developments of gasphase investigations into the structures of small gold clusters and their complexes with the proto-typical reactants $\mathrm{CO}$ and $\mathrm{O}_{2}$. The structural motifs adopted by the clusters are extremely unusual (when compared for example to transition metal clusters) with extended two-dimensional flake structures, hollow cages and tetrahedra being observed. The potential energy surfaces for these clusters provide evidence for structural isomerism and fluxionality even at reduced temperatures.

The reactivity patterns of the gold clusters with $\mathrm{O}_{2}$ can be largely rationalized by simple electron counting metrics, but structural aspects, i.e. the presence of low-coordinated $\mathrm{Au}$ atoms, are more relevant for the reactivity towards carbon monoxide. Whilst these metrics are not perfect they do allow for an initial understanding of the underlying features of gold clusters which encourage the catalytic $\mathrm{CO}$ oxidation. Again, in the characterization of the gold cluster complexes evidence has been presented for the importance of structural isomerism and fluxionality, which may act as a driving force for reactivity in some cases.

Generally the systems investigated require computational support for assignment of the experimental findings. Thereby, these studies on gold clusters highlight the vital interplay between gas-phase experimental characterization and accurate quantum chemical calculations. As more detailed experimental results, e.g. spectroscopic data, become available, greater demands are placed on the supporting theory. For example, the $2 \mathrm{D}$ to $3 \mathrm{D}$ transition in anionic gold clusters that is experimentally found to occur at $\mathrm{Au}_{12}$ [4] had been a puzzle for theory [51] but can now be correctly described thanks to more recent developments in density functional theory which are able to handle dispersion interactions more accurately $[5,62,130]$.

\section{Acknowledgments}

We gratefully acknowledge the contributions from all authors of our original papers, the "Stichting voor Fundamental Onderzoek der Materie (FOM)" in providing beam time on FELIX and the skilful assistance of the FELIX staff, in particular A.F.G. van der Meer and B. Redlich. This work is supported by the Max Planck Society, the Cluster of Excellence "Unifying Concepts in Catalysis" coordinated by the Technical University Berlin and funded by the Deutsche Forschungsgemeinschaft (DFG) and through the DFG within the research unit FOR 1282 (FI 893/4). We thank G. Meijer for his continued support. 


\section{References}

1. Yoon B, Häkkinen H, Landman U, Wörz A S, Antonietti J-M, Abbet S, Judai K and Heiz U (2005) Science 307:403

2. Molina L M and Hammer B (2005) J Catal 233:399

3. Lin X, Yang B, Benia H-M, Myrach P, Yulikov M, Aumer A, Brown M A, Sterrer M, Bondarchuk O, Kieseritzky E, Rocker J, Risse T, Gao H-J, Nilius N and Freund H-J (2010) J Am Chem Soc 132:7745

4. Furche F, Ahlrichs R, Weis P, Jacob C, Gilb S, Bierweiler T and Kappes M M (2002) J Chem Phys 117:6982

5. Johansson M P, Lechtken A, Schooss D, Kappes M M and Furche F (2008) Phys Rev A 77:053202

6. Huang W and Wang L-S (2009) Phys Rev Lett 102:153401

7. Häkkinen H, Yoon B, Landman U, Li X, Zhai H-J and Wang L-S (2003) J Phys Chem A 107:6168

8. Bulusu S, Li X, Wang L S and Zeng X C (2006) Proc Natl Acad Sci 103:8326

9. Li J, Li X, Zhai H-J and Wang L-S (2003) Science 299:864

10. Huang W, Bulusu S, Pal R, Zeng X C and Wang L-S (2009) ACS Nano 3:1225

11. Woodham A P, Meijer G and Fielicke A (2012) Angew Chem Int Ed 51:4444

12. Woodham A P, Meijer G and Fielicke A (2013) J Am Chem Soc 135:1727

13. Cox D M, Brickman R, Creegan K and Kaldor A (1991) Z Phys D - At, Mol Clusters 19:353

14. Kim Y D, Ganteför G, Sun Q and Jena P (2004) Chem Phys Lett 396:69

15. Huang W, Zhai H-J and Wang L-S (2010) J Am Chem Soc 132:4344

16. Lang S M, Bernhardt T M, Barnett R N and Landman U (2011) J Phys Chem C $115: 6788$

17. Socaciu L D, Hagen J, Bernhardt T M, Wöste L, Heiz U, Häkkinen H and Landman U (2003) J Am Chem Soc 125:10437

18. Schooss D, Weis P, Hampe O and Kappes M M (2010) Philos Trans R Soc, A 368:1211

19. Wang L-S (2010) Phys Chem Chem Phys 12:8694

20. Wang L-M and Wang L-S (2012) Nanoscale 4:4038

21. Bernhardt T M (2005) Int J Mass spectrom 243:1

22. Krückeberg S, Schooss D, Maier-Borst M and Parks J H (2000) Phys Rev Lett 85:4494

23. Maier-Borst M, Cameron D B, Rokni M and Parks J H (1999) Phys Rev A 59:R3162

24. von Helden G, Hsu M-T, Kemper P R and Bowers M T (1991) J Chem Phys 95:3835

25. Siegbahn K (1982) Rev Mod Phys 54:709

26. Leopold D G, Ho J and Lineberger W C (1987) J Chem Phys 86:1715

27. Neumark D M (2008) J Phys Chem A 112:13287

28. Yang Z, Leon I and Wang L-S (2013) J Chem Phys 139:021106

29. Gruene P, Rayner D M, Redlich B, van der Meer A F G, Lyon J T, Meijer G and Fielicke A (2008) Science 321:674

30. Onida G, Reining L and Rubio A (2002) Rev Mod Phys 74:601 
31. Schweizer A, Weber J M, Gilb S, Schneider H, Schooss D and Kappes M M (2003) J Chem Phys 119:3699

32. Collings B A, Athanassenas K, Lacombe D, Rayner D M and Hackett P A (1994) J Chem Phys 101:3506

33. Gilb S, Jacobsen K, Schooss D, Furche F, Ahlrichs R and Kappes M M (2004) J Chem Phys 121:4619

34. Gloess A N, Schneider H, Weber J M and Kappes M M (2008) J Chem Phys 128:114312

35. Collings B A, Athanassenas K, Rayner D M and Hackett P A (1993) Z Phys D - At, Mol Clusters 26:36

36. Lecoultre S, Rydlo A, Felix C, Buttet J, Gilb S and Harbich W (2011) J Chem Phys 134:074302

37. Hopkins W S, Woodham A P, Plowright R J, Wright T G and Mackenzie S R (2010) J Chem Phys 132:214303

38. Hopkins W S, Woodham A P, Plowright R J, Wright T G and MacKenzie S R (2011) J Chem Phys 134:094311

39. Bekkerman A, Kolodney E, von Helden G, Sartakov B, van Heijnsbergen D and Meijer G (2006) J Chem Phys 124:184312

40. Haertelt M, Lapoutre V J F, Bakker J M, Redlich B, Harding D J, Fielicke A and Meijer G (2011) J Phys Chem Lett 2:1720

41. Asmis K R, Fielicke A, Helden G v and Meijer G (2007) In D. P. Woodruff (eds) Atomic Clusters: From Gas Phase to Deposited Elsevier, Amsterdam, Netherlands, pp 327371 (chap. 8)

42. Oepts D, van der Meer A F G and van Amersfoort P W (1995) Infrared Phys Technol $36: 297$

43. Fielicke A, Kirilyuk A, Ratsch C, Behler J, Scheffler M, von Helden G and Meijer G (2004) Phys Rev Lett 93:023401

44. Fielicke A, von Helden G and Meijer G (2005) Eur Phys J D 34:83

45. Pyykkö P (2004) Angew Chem Int Ed 43:4412

46. Pyykkö P (2005) Inorg Chim Acta 358:4113

47. Pyykkö P (2008) Chem Soc Rev 37:1967

48. Weis P, Bierweiler T, Vollmer E and Kappes M M (2002) J Chem Phys 117:9293

49. Gilb S, Weis P, Furche F, Ahlrichs R and Kappes M M (2002) J Chem Phys 116:4094

50. Xing X, Yoon B, Landman U and Parks J H (2006) Phys Rev B 74:165423

51. Koskinen P, Häkkinen H, Huber B, von Issendorff B and Moseler M (2007) Phys Rev Lett 98:015701

52. Assadollahzadeh B and Schwerdtfeger P (2009) J Chem Phys 131:064306

53. Häkkinen H (2008) Chem Soc Rev 37:1847

54. Li X-B, Wang H-Y, Yang X-D, Zhu Z-H and Tang Y-J (2007) J Chem Phys 126:084505

55. Olson R M, Varganov S, Gordon M S, Metiu H, Chretien S, Piecuch P, Kowalski K, Kucharski S A and Musial M (2005) J Am Chem Soc 127:1049

56. Huang W and Wang L-S (2009) Phys Chem Chem Phys 11:2663 
57. Huang W, Pal R, Wang L-M, Zeng X C and Wang L-S (2010) J Chem Phys 132:054305

58. Pal R, Wang L-M, Huang W, Wang L-S and Zeng X C (2011) J Chem Phys 134:054306

59. Wang L-M, Pal R, Huang W, Zeng X C and Wang L-S (2010) J Chem Phys 132:114306

60. Lechtken A, Neiss C, Stairs J and Schooss D (2008) J Chem Phys 129:154304

61. Ghiringhelli L M, Gruene P, Lyon J T, Rayner D M, Meijer G, Fielicke A and Scheffler M (2013) New J Phys 15:083003

62. Beret E C, Ghiringhelli L M and Scheffler M (2011) Faraday Discuss 152:153

63. Pyykkö P and Runeberg N (2002) Angew Chem Int Ed 41:2174

64. Koyasu K, Naono Y, Akutsu M, Mitsui M and Nakajima A (2006) Chem Phys Lett 422:62

65. Kiran B, Li X, Zhai H-J and Wang L-S (2006) J Chem Phys 125:133204

66. Li X, Kiran B and Wang L-S (2005) J Phys Chem A 109:4366

67. Kiran B, Li X, Zhai H-J, Cui L-F and Wang L-S (2004) Angew Chem Int Ed 43:2125

68. Jena N K, Chandrakumar K R S and Ghosh S K (2011) J Phys Chem Lett 2:1476

69. Neukermans S, Janssens E, Tanaka H, Silverans R E and Lievens P (2003) Phys Rev Lett 90:033401

70. Janssens E, Tanaka H, Neukermans S, Silverans R E and Lievens P (2003) New J Phys $5: 46.1$

71. Tanaka H, Neukermans S, Janssens E, Silverans R E and Lievens P (2003) J Am Chem Soc 125:2862

72. Wang L-M, Pal R, Huang W, Zeng X C and Wang L-S (2009) J Chem Phys 130:051101

73. Wang L-M, Bai J, Lechtken A, Huang W, Schooss D, Kappes M M, Zeng X C and Wang L-S (2009) Phys Rev B 79:033413

74. Wang L-M, Bulusu S, Zhai H-J, Zeng X-C and Wang L-S (2007) Angew Chem Int Ed 46:2915

75. Wang L-M, Bulusu S, Huang W, Pal R, Wang L-S and Zeng X C (2007) J Am Chem Soc 129:15136

76. Lin L, Höltzl T, Gruene P, Claes P, Meijer G, Fielicke A, Lievens P and Nguyen M T (2008) ChemPhysChem 9:2471

77. Lin L, Claes P, Gruene P, Meijer G, Fielicke A, Nguyen M T and Lievens P (2010) ChemPhysChem 11:1932

78. Lang S M and Bernhardt T M (2012) Phys Chem Chem Phys 14:9255

79. Haruta M, Kobayashi T, Sano H and Yamada N (1987) Chem Lett 16:405

80. Haruta M, Yamada N, Kobayashi T and Iijima S (1989) J Catal 115:301

81. Bond G C and Thompson D T (1999) Cat Rev - Sci Eng 41:319

82. Mikami Y, Dhakshinamoorthy A, Alvaro M and García H (2013) Catal Sci Technol $3: 58$

83. Sanchez A, Abbet S, Heiz U, Schneider W D, Häkkinen H, Barnett R N and Landman U (1999) J Phys Chem A 103:9573

84. Mars P and van Krevelen D W (1954) Chem Eng Sci 3, Supplement 1:41 
85. Kimble M L, Moore N A, Johnson G E, Castleman A W, Jr., Burgel C, Mitric R and Bonacic-Koutecky V (2006) J Chem Phys 125:204311

86. Kimble M L and Castleman A W (2004) Int J Mass spectrom 233:99

87. Kimble M L, Moore N A, Castleman A W, Bürgel C, Mitrić R and Bonačić-Koutecký V (2007) Eur Phys J D 43:205

88. Johnson G E, Reilly N M, Tyo E C and Castleman A W (2008) J Phys Chem C 112:9730

89. Kim Y D, Fischer M and Ganteför G (2003) Chem Phys Lett 377:170

90. Salisbury B E, Wallace W T and Whetten R L (2000) Chem Phys 262:131

91. Lee T H and Ervin K M (1994) J Phys Chem 98:10023

92. Walter M and Hakkinen H (2006) Phys Chem Chem Phys 8:5407

93. Holleman A F and Wiberg E (1995) Lehrbuch der Anorganischen Chemie de Gruyter Berlin; New York

94. Stolcic D, Fischer M, Ganteför G, Kim Y D, Sun Q and Jena P (2003) J Am Chem Soc 125:2848

95. Pal R, Wang L-M, Pei Y, Wang L-S and Zeng X C (2012) J Am Chem Soc 134:9438

96. Mills G, Gordon M S and Metiu H (2002) Chem Phys Lett 359:493

97. Roldan A, Ricart J M, Illas F and Pacchioni G (2010) Phys Chem Chem Phys 12:10723

98. Franceschetti A, Pennycook S J and Pantelides S T (2003) Chem Phys Lett 374:471

99. Fernández E M, Ordejón P and Balbás L C (2005) Chem Phys Lett 408:252

100. Boronat $\mathrm{M}$ and Corma A (2010) Dalton Trans 39:8538

101. Lang S M, Bernhardt T M, Barnett R N, Yoon B and Landman U (2009) J Am Chem Soc 131:8939

102. Blyholder G (1964) J Phys Chem 68:2772

103. Hammer B and Nørskov J K (1995) Nature 376:238

104. Hammer B, Morikawa Y and Nørskov J K (1996) Phys Rev Lett 76:2141

105. Fielicke A, Gruene P, Meijer G and Rayner D M (2009) Surf Sci 603:1427

106. Sterrer M, Yulikov M, Fischbach E, Heyde M, Rust H-P, Pacchioni G, Risse T and Freund H-J (2006) Angew Chem Int Ed 45:2630

107. Balteanu I, Balaj O P, Fox B S, Beyer M K, Bastl Z and Bondybey V E (2003) Phys Chem Chem Phys 5:1213

108. Hagen J, Socaciu L D, Heiz U, Bernhardt T M and Wöste L (2003) Eur Phys J D $24: 327$

109. Neumaier M, Weigend F, Hampe O and Kappes M M (2005) J Chem Phys 122:104702

110. Neumaier M, Weigend F, Hampe O and Kappes M M (2008) Faraday Discuss 138:393

111. Fielicke A, von Helden G, Meijer G, Pedersen D B, Simard B and Rayner D M (2005)

J Am Chem Soc 127:8416

112. Fielicke A, von Helden G, Meijer G, Simard B and Rayner D M (2005) J Phys Chem B 109:23935

113. Yang X-F, Wang Y-L, Zhao Y-F, Wang A-Q, Zhang T and Li J (2010) Phys Chem Chem Phys 12:3038 
114. Pal R, Huang W, Wang Y-L, Hu H-S, Bulusu S, Xiong X-G, Li J, Wang L-S and Zeng X C (2011) J Phys Chem Lett 2288

115. Zhai H-J, Kiran B, Dai B, Li J and Wang L-S (2005) J Am Chem Soc 127:12098

116. Zhai H-J, Pan L-L, Dai B, Kiran B, Li J and Wang L-S (2008) J Phys Chem C 112:11920

117. Zhai H-J and Wang L-S (2005) J Chem Phys 122:051101

118. Wallace W T and Whetten R L (2000) J Phys Chem B 104:10964

119. Xie Y, Dong F and Bernstein E R (2011) Catal Today 177:64

120. Lupinetti A J, Fau S, Frenking G and Strauss S H (1997) J Phys Chem A 101:9551

121. Fielicke A, von Helden G, Meijer G, Pedersen D B, Simard B and Rayner D M (2006) J Chem Phys 124:194305

122. Fielicke A, von Helden G, Meijer G, Simard B and Rayner D M (2005) Phys Chem Chem Phys 7:3906

123. Jackschath C, Rabin I and Schulze W (1992) Ber Bunsen-Ges Phys Chem 96:1200

124. Veldeman N, Lievens P and Andersson M (2005) J Phys Chem A 109:11793

125. Fielicke A, Gruene P, Meijer G and Rayner D M Unpublished Data

126. Wallace W T and Whetten R L (2002) J Am Chem Soc 124:7499

127. Wallace W T, Wyrwas R B, Whetten R L, Mitrić R and Bonačić-Koutecký V (2003) J Am Chem Soc 125:8408

128. Hagen J, Socaciu L D, Elijazyfer M, Heiz U, Bernhardt T M and Wöste L (2002) Phys Chem Chem Phys 4:1707

129. Häkkinen H and Landman U (2001) J Am Chem Soc 123:9704

130. Mantina M, Valero R and Truhlar D G (2009) J Chem Phys 131:064706 